\title{
A robust model for rapidly varying flows over movable bottom with suspended and bedload transport: modelling and numerical approach
}

\author{
González-Aguirre, J. C. ${ }^{\mathrm{a}, *}$, Castro, M.J. ${ }^{\mathrm{a}}$, Morales de Luna, T. ${ }^{\mathrm{b}}$ \\ ${ }^{a}$ Dpto. Análisis Matemático, Universidad de Málaga, Spain \\ ${ }^{b}$ Dpto. Matemáticas, Universidad de Córdoba, Spain
}

\begin{abstract}
We propose a coupled model for suspended and bedload sediment transport in the shallow water framework. The model is deduced under hydrostatic pressure assumptions and will not assume any Bossinesq hypothesis. The numerical resolution is carried out in a segregated way. First the underlying system of conservation laws is solved by using a first order path-conservative Riemann solver. Then, the source terms corresponding with the erosion and depositions rates are approximated in a semi-implicit way. The final scheme preserves the positivity of the density. Several numerical experiments were carried out in order to validate the model and the numerical scheme. The results obtained are in good agreement with the experimental data.
\end{abstract}

Keywords: Sediment transport, Shallow water Exner models, bedload, suspended load, finite volume solvers, well-balanced schemes

\section{Introduction}

In geophysics and earth sciences the term fluvial is used to refer to the processes associated with the rivers or streams and erosion or deposits and the morphology created by them. The sediment is transported by the river current

\footnotetext{
*Dpto. Análisis Metemático, Universidad de Málaga, Spain

Email address: jcga@uma.es (González-Aguirre, J. C. )
} 
5 as suspended load (finer fractions carried by the flow) and bed load (coarse fractions which move close to the bottom rolling, jumping and sliding), see [1].

Knowledge of sediment transport can be applied in civil engineering, for example, to plan the extended life of a dam forming a reservoir. The sediment transport can be applied to solve environmental engineering problems, for example, it is important in providing habitat for fish in river or estuaries, sustaining a hygienic stream ecosystem. The sediment carried by a river is deposed downstream reducing the capacity of the river at that area, which can results in frequent floods at outskirts of the river.

The study of sediment transport focuses on understanding the relationship that exists between the movement of water and the movement of sedimentary materials. A first approach to model bedload transport is to couple the shallow water equations with the so-called Exner equation (See [2]). This equation depends on the empirical definition of the solid transport flux for the bedload transport. Several formulations have been proposed, see for instance [3, 4, 5, 6] among others. This approach has been extensively used to describe bedload transport, see [7, 8, 9, 10, 11, 12, 13, among many others.

Suspended load accounts for sedimentary particles which have been eroded from the bottom and remain floating on the current for some time until they are sedimented afterwards. In [14] was proposed a model with full considerations of 25 the mass and momentum transfers due to the sediment exchange between the bottom and the water. This model includes the mass and momentum conservation equation for the water sediment mixture and the mass conservation of the sediment, but the bed deformation was not taken into account. In [15, 16] the mass conservation for the bed material was considered in order to incorporate the effects of the bed deformation on the fluid flux. Similar models have been used in [17, 18, 19, 20, 21, 22, 23. All of these models are based on the assumption of hydrostatic pressure. A more recent work [24] introduces a non-hydrostatic model for sediment transport.

One of the assumptions that is usually done in sediment transport models 35 is the Boussinesq hypothesis. This means that spatial variations of the density 
of the water sediment mixture are ignored in the convective part and are only taken into account into the pressure term. This assumption is valid when the variations of density are small. This can be inaccurate in many situations. One objective of this work is to deduce a general mathematical model for sediment

40 transport without such assumption. This model will be proposed under the shallow water framework and aims at accurately describing both bedload and suspended sediment transport. Up to our knowledge, sediment transport models based on the shallow water framework usually use Boussinesq hypothesis which makes the numerical computations easier. Nevertheless this may result in inaccurate results in situations where we are far from this assumption.

A second objective of this paper is to described an efficient and robust numerical approximation of the model. This will be done by using a a two-step algorithm, following similar ideas as in [25, 26, 27, 28]. During the first step, the hyperbolic part of the system will be solved using a path-conservative wellbalanced numerical scheme (See [29, 30]). Moreover, both bedload and suspended load will be solved without decoupling the system. As shown in 31, a splitting technique may result into instabilities. During the second step, erosiondeposition terms are solved using a novel semi-implicit approach. This will allow to guarantee the positivity preserving of density and water-thickness.

55 This work is structured as follows: First, the mathematical model is deduced. In Section 3, a general compact formulation will be presented as well as a study of the hyperbolicity of the model and the characterization of some relevant stationary solutions. The numerical scheme to be used will be introduced in Section 4. In Section 5 , the extension to the two-dimensional case will be briefly shown. Finally, in Section 6, several numerical experiments are carry out to validate the model and the numerical approach used.

\section{Deduction of mathematical model}

We consider a water flow over movable bottom $b(\mathbf{x}, t)$ that contains suspended particles of diameter $d$ and density $\rho_{s}$. The movement of water is 
characterized by the time evolution of both the water depth $h(\mathbf{x}, t)$ and the velocity $\mathbf{u}(\mathbf{x}, t)=(u(\mathbf{x}, t), v(\mathbf{x}, t), w(\mathbf{x}, t))$ where $\mathbf{x} \in \mathbb{R}^{3}$. We shall denote by $\eta(\mathbf{x}, t)=h(\mathbf{x}, t)+b(\mathbf{x}, t)$ the free surface of the fluid and by $\rho_{0}$ the density of water. The evolution of the bottom, assumed with porosity $p$, is the result of two processes: erosion-deposition of the bottom and bedload transport. The first one corresponds to a transfer of particles between the bottom and the suspended load in the fluid. This process gives a mixture of water and sediment with density $\rho(\mathbf{x}, t)$ and volumetric sediment concentration $c(\mathbf{x}, t)$. The erosion and deposition fluxes will be denoted by $F_{e}(\mathbf{x}, t)$ and $F_{d}(\mathbf{x}, t)$ respectively. The second process, called bedload transport, takes into account sedimentary 75 particles which move along the bottom by rolling, sliding and jumping. This transport is measured by the bedload discharge $q_{b}(\mathbf{x}, t)$. A one dimensional sketch of this phenomenon is shown in Figure 1.

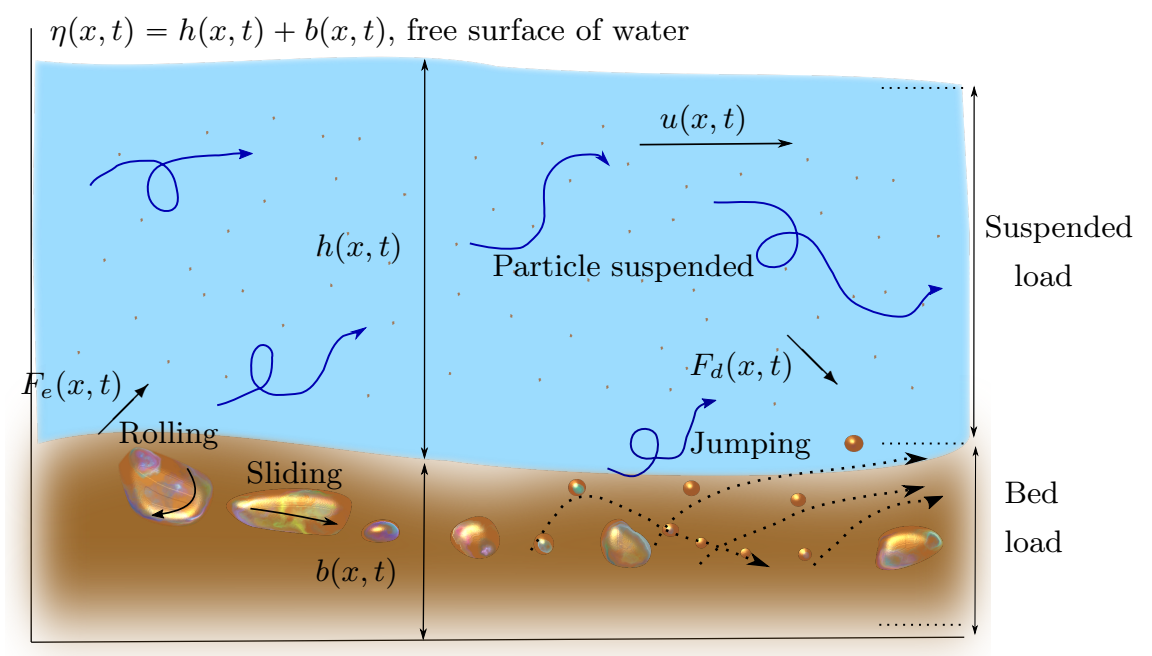

Figure 1: Sketch of bed load and suspended-load sediment transport.

The mathematical model to be used is based on the Navier-Stokes equations:

$$
\begin{gathered}
\partial_{t} \rho+\operatorname{div}(\rho \mathbf{u})=0 . \\
\operatorname{div}(\rho \mathbf{u})=0
\end{gathered}
$$




$$
\partial_{t}(\rho \mathbf{u})+\operatorname{div}(\rho \mathbf{u} \otimes \mathbf{u})=-\nabla p+\nabla \cdot \mathcal{T}+\rho \mathbf{g},
$$

where $p(\mathbf{x}, t)$ denotes the instantaneous pressure. The density of the mixture is given by

$$
\rho(\mathbf{x}, t)=\rho_{0}+c(\mathbf{x}, t)\left(\rho_{s}-\rho_{0}\right),
$$

the vector $\mathbf{g}=(0,0,-g)^{t}$ is the gravity and

$$
\mathcal{T}=\mu\left(\nabla \mathbf{u}+(\nabla \mathbf{u})^{t}\right)
$$

is the stress tensor, whith $\mu$ the viscosity coefficient. We shall consider the kinematic boundary conditions

$$
\begin{gathered}
\partial_{t} \eta+\left.u\right|_{z=\eta} \partial_{x} \eta-\left.w\right|_{z=\eta}=0, \\
\partial_{t} b+\left.u\right|_{z=b} \partial_{x} b-\left.w\right|_{z=b}=-\widetilde{\phi}_{b},
\end{gathered}
$$

where $\widetilde{\phi}_{b}=\left(1+\left(\partial_{x} b\right)^{2}\right)^{1 / 2} \varphi^{b}$. Here $\varphi^{b}$ is the normal erosion-deposition rate.

For the sake of simplicity, we shall neglect the variations on the horizontal direction $y$. Nevertheless everything said here is easily extended otherwise.

\subsection{Vertical integration}

In this section we shall consider that the horizontal effects are predominant with respect the vertical ones, and therefore, we suppose that the vertical variations of the velocity are small. Under this assumptions we proceed to do a vertical integration of the equations. We shall assume that the fluid is incompressible, that is,

$$
\operatorname{div} \mathbf{u}=0
$$

By integrating from $z=b$ up to $z=\eta$ equation (2.1) we get

$$
\int_{b}^{\eta} \partial_{t} \rho d z+\int_{b}^{\eta} \partial_{x}(\rho u) d z=\left.\rho w\right|_{z=b}-\left.\rho w\right|_{z=\eta} .
$$

Using 2.6 and (2.7), equation 2.9 takes the form

$$
\partial_{t} \int_{b}^{\eta} \rho d z+\partial_{x} \int_{b}^{\eta} \rho u d z=\rho \tilde{\phi}_{b}
$$


The momentum conservation equation $(2.3)$ written in terms of its components reads

$$
\begin{aligned}
\partial_{t}(\rho u)+\partial_{x}\left(\rho u^{2}\right)+\partial_{z}(\rho u w) & =-\partial_{x} p+\mu\left(2 \partial_{x x} u+\partial_{z} \widetilde{\tau}\right), \\
\partial_{t}(\rho w)+\partial_{x}(\rho w u)+\partial_{z}\left(\rho w^{2}\right) & =-\partial_{z} p+\mu\left(\partial_{x} \widetilde{\tau}+2 \partial_{z z} w\right)-\rho g,
\end{aligned}
$$

where $\widetilde{\tau}=\partial_{x} w+\partial_{z} u$. As we have said, we shall assume that vertical effects in 2.11) and 2.12 are negligible. In particular, we shall assume that the pressure is hydrostatic so that 2.12 reduces to

$$
\partial_{z} p=-\rho g
$$

Therefore, integrating 2.11 from $b$ up to $\eta$ and assuming $\partial_{x x} u \approx 0$ we get

$$
\begin{aligned}
\int_{b}^{\eta} \partial_{t}(\rho u) d z+\int_{b}^{\eta} \partial_{x}\left(\rho u^{2}\right) d z+\int_{b}^{\eta} \partial_{x} p d z \\
\quad=\left.\left.\rho u\right|_{z=b} w\right|_{z=b}-\left.\left.\rho u\right|_{z=\eta} w\right|_{z=\eta}+\mu\left(\left.\widetilde{\tau}\right|_{z=\eta}-\left.\widetilde{\tau}\right|_{z=b}\right) .
\end{aligned}
$$

Taking into account kinematic boundary condition, 2.14 becomes

$$
\begin{aligned}
\partial_{t} \int_{b}^{\eta} \rho u d z+\partial_{x} \int_{b}^{\eta} \rho u^{2} d z+\partial_{x} \int_{b}^{\eta} p d z & =\left.\rho u\right|_{z=b} \tilde{\phi}_{b} \\
& +\mu\left(\left.\widetilde{\tau}\right|_{z=\eta}-\left.\widetilde{\tau}\right|_{z=b}\right) .
\end{aligned}
$$

Definition 2.1. For any function $f(x, z, t)$, we define its mean on the vertical direction as

$$
\bar{f}=\frac{1}{h} \int_{b}^{\eta} f d z .
$$

The fluctuating part $\hat{f}$ of $f$ relative to the mean is defined as

$$
\widehat{f}=f-\bar{f} .
$$

From definition 2.1 we can see that, given two functions $f_{1}, f_{2}$ we get

$$
h \overline{f_{1}} \overline{f_{2}}+\int_{b}^{\eta} \widehat{f_{1}} \widehat{f_{2}} d z=\int_{b}^{\eta} f_{1} f_{2} d z .
$$

Using definition 2.1 and 2.18, equation 2.10 writes

$$
\partial_{t}(h \bar{\rho})+\partial_{x}\left(h \bar{\rho} \bar{u}+\int_{b}^{\eta} \widehat{\rho} \widehat{u} d z\right)=\rho \widetilde{\phi}_{b} .
$$


From 2.13 we get

$$
p(x, z, t)=g \bar{\rho}(\eta-z)+g \int_{z}^{\eta} \hat{\rho} d z^{\prime}
$$

therefore

$$
\partial_{x} p(x, z, t)=g \bar{\rho} \partial_{x} \eta+g(\eta-z) \partial_{x} \bar{\rho}+g \partial_{x} \int_{z}^{\eta} \hat{\rho} d z^{\prime} .
$$

Finally

$$
\int_{b}^{\eta} \partial_{x} p d z=g h \bar{\rho} \partial_{x} \eta+g \frac{h^{2}}{2} \partial_{x} \bar{\rho}+g \int_{b}^{\eta}\left(\partial_{x} \int_{z}^{\eta} \hat{\rho} d z^{\prime}\right) d z .
$$

Taking into account 2.22, equation 2.15 takes the form

$$
\begin{aligned}
\partial_{t} \int_{b}^{\eta}(\rho u) d z & +\partial_{x} \int_{b}^{\eta}\left(\rho u^{2}\right) d z+g h \bar{\rho} \partial_{x} \eta+g \frac{h^{2}}{2} \partial_{x} \bar{\rho}+g \int_{b}^{\eta}\left(\partial_{x} \int_{z}^{\eta} \hat{\rho} d z^{\prime}\right) d z . \\
& =\mu\left(\left.\widetilde{\tau}\right|_{z=\eta}-\left.\widetilde{\tau}\right|_{z=b}\right)+\left.\rho u\right|_{z=b} \widetilde{\phi}_{b} .
\end{aligned}
$$

Using definition 2.1 and equation 2.17) we get the averaged momentum equation

$$
\begin{aligned}
\partial_{t}(h \bar{\rho} \bar{u}) & +\partial_{x} h\left(\bar{\rho} \bar{u}^{2}\right)+\partial_{x}\left(g \frac{h^{2}}{2} \bar{\rho}\right)=-g h \bar{\rho} \partial_{x} b \\
& +\mu\left(\left.\widetilde{\tau}\right|_{z=\eta}-\left.\widetilde{\tau}\right|_{z=b}\right)+\left.\rho u\right|_{z=b} \widetilde{\phi}_{b}-\partial_{t} \int_{b}^{\eta} \widehat{\rho} \widehat{u} d z \\
& -\partial_{x} \int_{b}^{\eta} \widehat{\rho} \widehat{u}^{2} d z-g \int_{b}^{\eta}\left(\partial_{x} \int_{z}^{\eta} \hat{\rho} d z^{\prime}\right) d z .
\end{aligned}
$$

We shall assume that the fluctuating parts are negligible and we consider that the tensor stress can be expressed in terms of $\bar{u}$, so that, we write

$$
\mu\left(\left.\widetilde{\tau}\right|_{z=\eta}-\left.\widetilde{\tau}\right|_{z=b}\right)=\tau(\bar{u}) .
$$

We shall denote

$$
\phi_{b}=c \widetilde{\phi}_{b}
$$

It is usual to express the velocity at surface, $\left.u\right|_{z=\eta}$ and bottom $\left.u\right|_{z=b}$, in terms of mean velocity $\bar{u}$. Entropy considerations leads us to assume $\left.u\right|_{z=\eta}=\bar{u}$ 
90 and $\left.u\right|_{z=b}=\frac{1}{2} \bar{u}$, see 32,33 . Therefore, equations 2.19 and 2.24 take the form

$$
\begin{aligned}
& \partial_{t}(h \bar{\rho})+\partial_{x}(h \bar{\rho} \bar{u})=\rho_{0} \tilde{\phi}_{b}+\phi_{b}\left(\rho_{s}-\rho_{0}\right) \\
& \partial_{t}(h \bar{\rho} \bar{u})+\partial_{x}\left(h \bar{\rho} \bar{u}^{2}\right)+\partial_{x}\left(g \frac{h^{2}}{2} \bar{\rho}\right)=-g h \bar{\rho} \partial_{x} b \\
&+\tau(\bar{u})+\bar{\rho} \frac{\bar{u}}{2} \phi_{b} .
\end{aligned}
$$

Henceforth we neglect the symbol -. Following [16], in order to take into account the bed deformation, the density of sediment $\rho_{s}$ in the source term is usually written in terms of the density of saturated bottom $\rho_{b}$ given by

$$
\rho_{b}=\rho_{0} p+\rho_{s}(1-p)
$$

therefore 2.27 becomes

$$
\partial_{t}(h \rho)+\partial_{x}(h \rho u)=\frac{\phi_{b} \rho_{b}}{1-p}+\rho_{0}\left(\tilde{\phi}_{b}-\frac{\phi_{b}}{1-p}\right) .
$$

The dynamic of bedload sediment transport is modeled using the Exner equation, see [34, 2]. This equation states that the time variation of the sediment layer over a certain volume is due to of the net variation of the solid transport through the boundaries of the volume. Exner equation reads

$$
\partial_{t} b+\frac{1}{1-p} \partial_{x} q_{b}=-\frac{1}{1-p} \phi_{b},
$$

where $q_{b}=q_{b}(h, h u)$ denotes the solid transport flux.

Let us consider the simple case where the sediment is confined in a closed domain, so that there is not exchange through boundaries. In particular, assume for simplicity the case where $u=0$. Then, one should get that the total sediment mass in the interior of the domain is preserved, that is

$$
\partial_{t}\left(h \rho+\rho_{b} b\right)=0
$$

Therefore from 2.30, 2.31 and 2.32 one should obtain

$$
\widetilde{\phi}_{b}-\frac{\phi_{b}}{1-p}=0
$$


From the integral averaged form of the continuity equation (2.1), taking into account that the density is transported and considering $(2.33)$, we get the volume conservation equation

$$
\partial_{t} h+\partial_{x}(h u)=\frac{\phi_{b}}{1-p} .
$$

Let us remark that the deduction of the model does not use Boussinesq hypothesis. The final model then writes

$$
\begin{gathered}
\partial_{t} h+\partial_{x}(h u)=\frac{\phi_{b}}{1-p}, \\
\partial_{t}(h \rho)+\partial_{x}(h \rho u)=\frac{\phi_{b} \rho_{b}}{1-p}, \\
\partial_{t}(h \rho u)+\partial_{x}\left(h \rho u^{2}\right)+\partial_{x}\left(g \frac{h^{2}}{2} \rho\right)=-g h \rho \partial_{x} b \\
+\tau(u)+\rho \frac{u}{2} \phi_{b}, \\
\partial_{t} b+\frac{1}{1-p} \partial_{x} q_{b}=-\frac{1}{1-p} \phi_{b},
\end{gathered}
$$

\subsection{Erosion and deposition effects}

To close the system the sediment exchange between the bottom and the water $\left(\phi_{b}\right)$ is determined from the rates of deposition $\left(F_{d}\right)$ and erosion $\left(F_{e}\right)$, namely

$$
\phi_{b}=F_{e}-F_{d} .
$$

\subsubsection{Deposition rate}

The deposition rate is described as the product of the settling velocity of sediment (drop velocity of the particle under water at rest) $v_{s}$, and the fractional concentration of suspension near by the bed $c_{b}$,

$$
F_{d}=v_{s} c_{b}
$$


In order to compute $c_{b}$ we shall use the formula proposed in [35], which states that

$$
\frac{c_{b}}{c}=0.4\left(\frac{d}{D_{s g}}\right)^{1.64}+1.64
$$

where $D_{s g}$ denotes the geometric mean size of the suspended sediment mixture and $d$ is the sediment diameter.

The settling velocity of sediment may be obtained experimentally or using the formulation proposed by Zhang and Xie [36, which has been used in several works like 20,23 .

$$
v_{s}=\sqrt{\left(\frac{13.95 \nu}{d}\right)^{2}+1.09 s g d}-\frac{13.95 \nu}{d},
$$

where $\nu$ denotes the kinematic viscosity of the water, $s=\frac{\rho_{s}}{\rho_{0}}-1$ is the relative density and $s g$ is the specific gravity of the submerged sediment.

\subsubsection{Erosion rate}

The erosion rate from the bottom is computed using the following expression

$$
F_{e}=v_{s} \mathcal{P} E_{s}
$$

The sediment entrainment coefficient $E_{s}$, is computed using the formulation developed by Garcia and Parker [37,

$$
E_{s}=\frac{1.3 \times 10^{-7} \mathcal{Z}^{5}}{1+4.3 \times 10^{-7} \mathcal{Z}^{5}}
$$

where

$$
\mathcal{Z}=\alpha_{1} \frac{\sqrt{c_{D}}|u|}{v_{s}} \mathcal{R}_{j}^{\alpha_{2}}
$$

and

$$
\mathcal{R}_{p}=\frac{\sqrt{s g d} d}{\nu}
$$

is the particle Reynold number. Several values have been proposed for the parameters $\left(\alpha_{1}, \alpha_{2}\right)$ as it can be seen in $32,35,38,39$. In this work we shall employ the values $(1,0.6)$ if $\mathcal{R}_{p}>2.36$ and $(0.586,1.23)$ when $\mathcal{R}_{p} \leqslant 2.36$. The 
constant $\mathcal{P}$ represents the volume fraction of the sediment in the bottom. In [40] is it said that if $\mathcal{P}$ represents the volumetric solids fraction of the sediment, then $1-\mathcal{P}$ denotes the porosity of the control volume.

\subsubsection{Friction term}

Following [16], the friction term is computed as

$$
\tau(u)=\rho u_{*}^{2}\left(1+r_{w}\right)
$$

where $u_{*}$ is the bed shear velocity and it is computed as 15 by

$$
u_{*}^{2}=\frac{g \mu_{f}^{2} u^{2}}{h^{1 / 3}},
$$

where $\mu_{f}$ is the dimensionless Manning coefficient. The term $r_{w}$ denotes the ratio of upper-interface resistance to bed resistance.

\subsubsection{Bed load discharge}

The computation of the bed load discharge $q_{b}$ is based on empirical laws. Some of the most used ones were proposed Meyer-Peter and Müller [3] , Grass [5], Van Rijn [6], among others. In this work we use the formulation proposed by Meyer-Peter and Müller, namely

$$
\frac{q_{b}}{Q}=8 \operatorname{sgn}(u)\left(\theta-\theta_{c}\right)_{+}^{3 / 2},
$$

where

$$
Q=d \sqrt{g s d}
$$

is the characteristic discharge, $\theta$ denotes the Shields parameter (ratio between agitating and stabilizing forces on a sediment grain in the bottom)

$$
\theta=\frac{u_{*}^{2}}{s g d}
$$

and $\theta_{c}$ is the critical Shields stress for incipient motion. 


\section{General formulation of the model}

125 vectorial form as

$$
\partial_{t} \mathbf{W}+\partial_{x} \mathbf{F}(\mathbf{W})+\mathbf{B}(\mathbf{W}) \partial_{x} \mathbf{W}=\mathbf{G}(\mathbf{W})+\mathbf{S}_{\tau}(\mathbf{W})
$$

where the vector of conservative variables takes the form $\mathbf{W}=(h, h \rho, h \rho u, b)^{t}$. The physical flux $\mathbf{F}$, the non-conservative product matrix $\mathbf{B}$ and the source terms, $\mathbf{G}$ (erosion and deposition) and $\mathbf{S}_{\tau}$ (friction term), have the followings form

$$
\begin{gathered}
\mathbf{F}(\mathbf{W})=\left(\begin{array}{c}
h u \\
h \rho u \\
h \rho u^{2} \\
\frac{q_{b}}{1-p}
\end{array}\right), \quad \mathbf{B}(\mathbf{W})=\left(\begin{array}{cccc}
0 & 0 & 0 & 0 \\
0 & 0 & 0 & 0 \\
\frac{g}{2} h \rho & \frac{g}{2} h & 0 & g h \rho \\
0 & 0 & 0 & 0
\end{array}\right), \\
\mathbf{G}(\mathbf{W})=\left(\begin{array}{c}
\frac{\phi_{b}}{1-p} \\
\frac{\phi_{b} \rho_{b}}{1-p} \\
\rho \frac{u}{2} \phi_{b} \\
-\frac{\phi_{b}}{1-p}
\end{array}\right), \quad \mathbf{S}_{\tau}=\left(\begin{array}{c}
0 \\
0 \\
\rho u_{*}^{2}\left(1+r_{w}\right) \\
0
\end{array}\right)
\end{gathered}
$$

System (3.1) may also be written in quasi-linear form as

$$
\partial_{t} \mathbf{W}+\mathcal{A}(\mathbf{W}) \partial_{x} \mathbf{W}=\mathbf{G}(\mathbf{W})+\mathbf{S}_{\tau}(\mathbf{W})
$$

where $\mathcal{A}(\mathbf{W})=\frac{\partial \mathbf{F}(\mathbf{W})}{\partial \mathbf{W}}+\mathbf{B}(\mathbf{W})$.

The problem to be solved can be formulated as follows. Given the initial condition $\mathbf{W}_{0}$, we need to compute the state $\mathbf{W}_{t}, t>0$ by solving the system (3.4) on $[0, \mathcal{L}]$, where $\mathcal{L}$ is the length of channel, subject to some boundary conditions. 


\subsection{Hyperbolic character of the system}

Given that the bed load discharge flux does not depend on the depth of bottom, we have $\partial_{b} q_{b}=0$. Let us be consider the matrix

$$
\mathcal{A}=\left(\begin{array}{cccc}
u & -\frac{u}{\rho} & \frac{1}{\rho} & 0 \\
0 & 0 & 1 & 0 \\
\frac{g}{2} h \rho & \frac{g}{2} h-u^{2} & 2 u & g h \rho \\
\alpha & \beta & \delta & 0
\end{array}\right),
$$

where

$$
\alpha=\partial_{h} q_{b}, \quad \beta=\partial_{h \rho} q_{b}, \quad \delta=\partial_{h \rho u} q_{b} .
$$

Following 31, bedload transport fluxes may be written as a function of the Shields parameter, that is,

$$
q_{b} \equiv \operatorname{sgn}(u) \Phi(\theta)
$$

for some function $\Phi$. In particular, if the Meyer-Peter\&Müller formula is used, we have $\Phi(\theta)=8 Q\left(\theta-\theta_{c}\right)_{+}^{3 / 2}$. Nevertheless everything said here may be generalized for any other solid transport flux formula.

Therefore, we get

$\alpha=\operatorname{sgn}(u) \Phi^{\prime}(\theta) \partial_{h} \theta, \quad \beta=\operatorname{sgn}(u) \Phi^{\prime}(\theta) \partial_{h \rho} \theta, \quad \delta=\operatorname{sgn}(u) \Phi^{\prime}(\theta) \partial_{h \rho u} \theta$.

Remark that from 2.50$)$ we have

$$
\partial_{h} \theta=-\frac{1}{6} \rho u \partial_{h \rho u} \theta, \quad \partial_{h \rho} \theta=-u \partial_{h \rho u} \theta .
$$

Therefore

$$
\alpha=-\frac{1}{6} \rho u \delta, \quad \beta=-u \delta .
$$

Some straightforward calculations show that the characteristic polynomial of $\mathcal{A}$ is

$$
p(\lambda)=-(u-\lambda)\left(\lambda^{3}+a_{2} \lambda^{2}+a_{1} \lambda+a_{0}\right),
$$


with

$$
\begin{aligned}
& a_{2}=-2 u \\
& a_{1}=-\left(g h \rho\left(\delta+\frac{1}{2 \rho}\right)+\left(\frac{g}{2} h-u^{2}\right)\right), \\
& a_{0}=-g h \rho\left(\beta+\frac{\alpha}{\rho}\right) .
\end{aligned}
$$

Thus, $u$ is an eigenvalue of the matrix $\mathcal{A}$. In order to prove the hyperbolicity of the system (3.4) we need to ensure that the cubic polynomial on the right hand side of 3.9 has three real roots. Remark that this polynomial may be written as

$$
P_{A}(\lambda)=f(\lambda)-d(\lambda)
$$

where

$$
\begin{gathered}
f(\lambda)=\lambda\left((u-\lambda)^{2}-g h\right), \\
d(\lambda)=g h \rho\left(\lambda-\frac{7}{6} u\right) \delta .
\end{gathered}
$$

This means that we have three real roots whenever the line $d(\lambda)$ intersects $f(\lambda)$ in three points. In other words, we need to ensure that the root of $d(\lambda)$, that ${ }_{145}$ is $\frac{7}{6} u$ lies between the roots $\alpha_{ \pm}$of $d_{ \pm}(\lambda)$, where $d_{ \pm}(\lambda)$ are the tangent straight lines to the curve $f(\lambda)$ and parallel to $d(\lambda)$. A sketch of this fact can be seen in Figure 2 ,

More explicitly, the lines $d_{ \pm}(\lambda)$ take the form

$$
d_{ \pm}(\lambda)=f\left(\lambda_{ \pm}\right)-g h \rho \delta\left(\lambda-\lambda_{ \pm}\right)
$$

where

$$
\lambda_{ \pm}=\frac{2 u+\sqrt{u^{2}+3 g h(1-\rho \delta)}}{3} .
$$

The roots of the straight lines $d_{ \pm}(\lambda)$ are given by

$$
\alpha_{ \pm}=\frac{f\left(\lambda_{ \pm}\right)}{g h \rho \delta}+\lambda_{ \pm}
$$

System (3.4) will be hyperbolic whenever the next relation is fulfilled:

$$
\alpha_{-}<\frac{7}{6} u<\alpha_{+}
$$




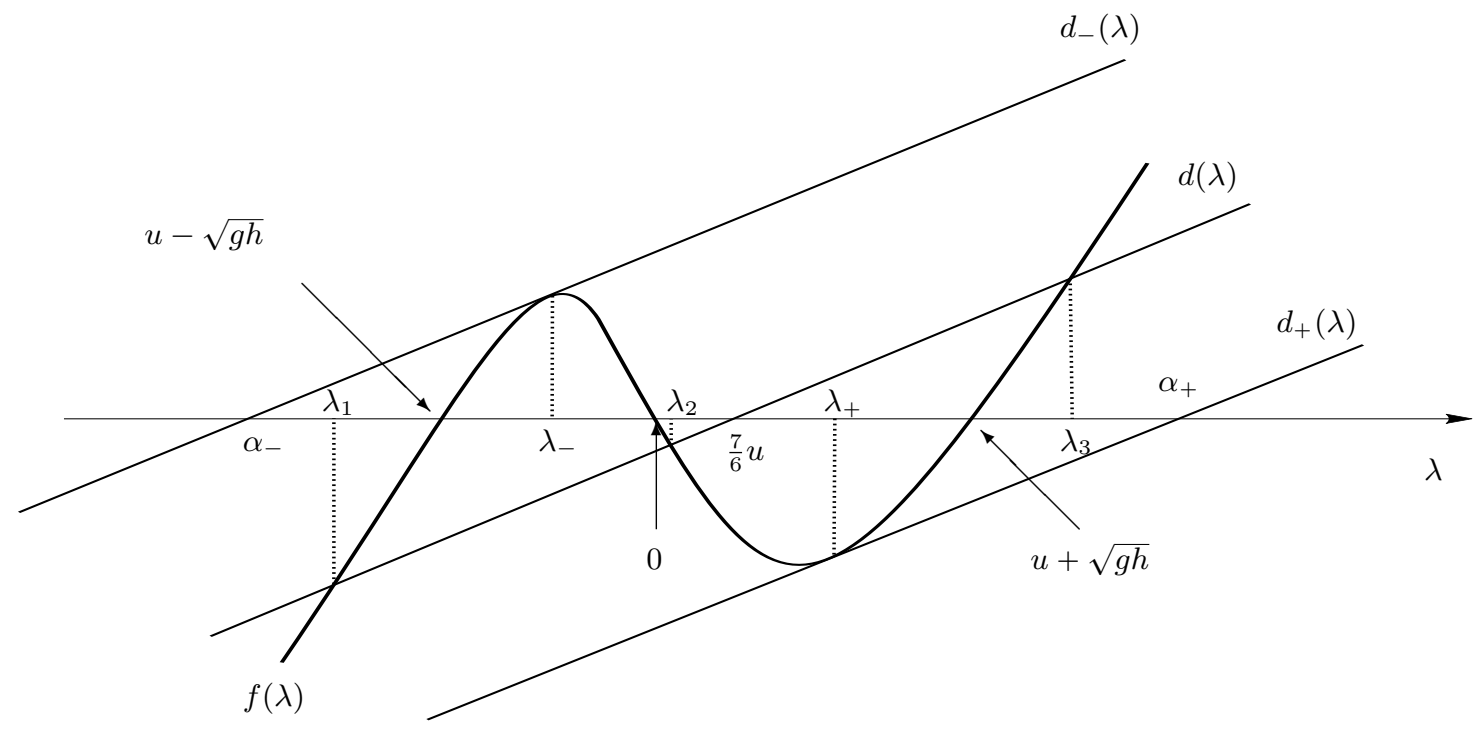

Figure 2: Sketch of the proof for the hyperbolicity.

Remark that a sufficient condition for the system to be hyperbolic is that $|u|<6 \sqrt{g h}$, which is in general true for physical situations. Indeed, one can ${ }_{150}$ check that in this case

$$
\alpha_{-}<u-\sqrt{g h}<\frac{7}{6} u<u+\sqrt{g h}<\alpha_{+} .
$$

In order to compute an approximation for characteristics velocities, we shall employ the following procedure, which is depicted in Figure 3

- Let us consider

$$
S_{-}=u-\sqrt{g h}, \quad S_{m i d}=0, \quad \text { and } \quad S_{+}=u+\sqrt{g h},
$$

the roots of (3.11).

- We compute the tangent straight line $l_{ \pm}(\lambda)$ to $f(\lambda)$ at point $\left(S_{ \pm}, 0\right)$

$$
l_{ \pm}(\lambda)=f^{\prime}(\lambda)\left(\lambda-S_{ \pm}\right) .
$$

- We solve the equation $l_{ \pm}(\lambda)=-d(\lambda)$, whose solution is

$$
\lambda_{ \pm}^{*}=\frac{f^{\prime}\left(S_{ \pm}\right) S_{ \pm}-\frac{7}{6} g h \rho u \delta}{f^{\prime}\left(S_{ \pm}\right)-g h \rho \delta} .
$$




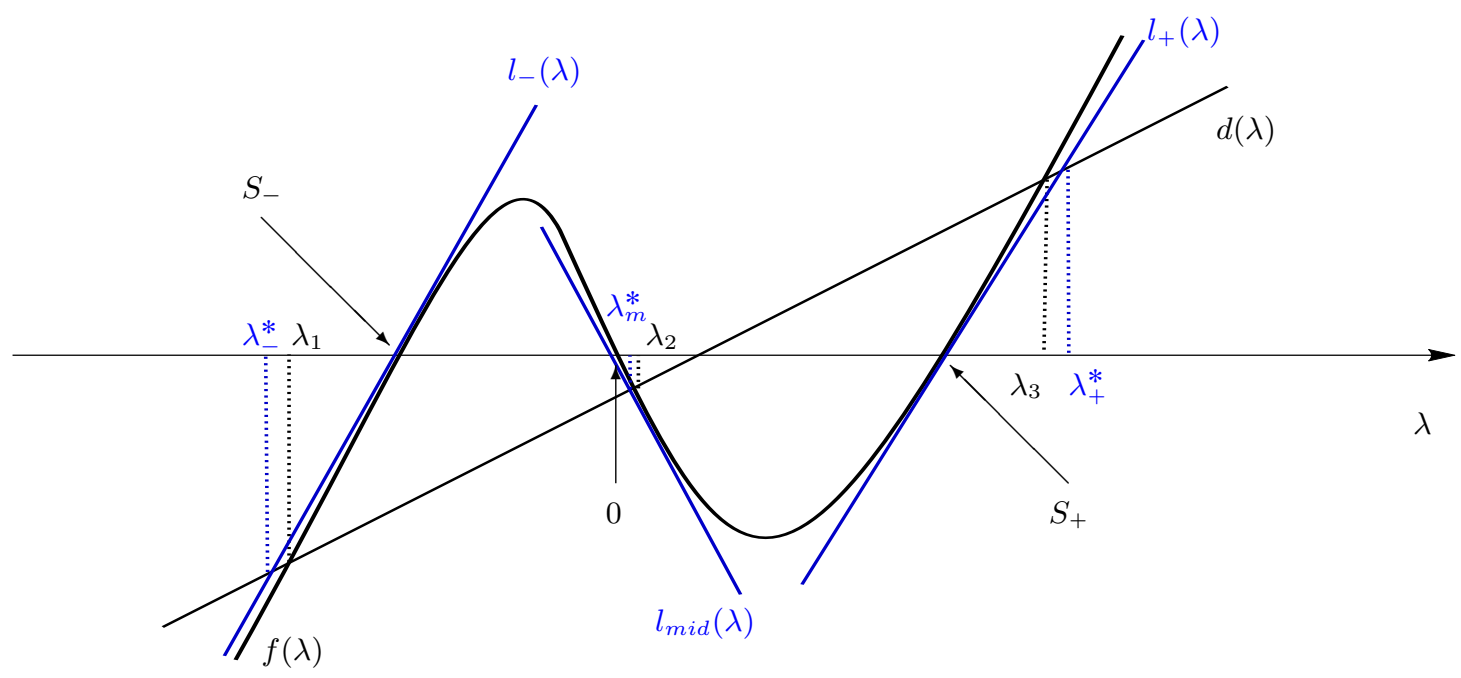

Figure 3: Sketch of the procedure to compute the characteristics velocities of 3.4.

- We compute the tangent straight line $l_{\text {mid }}$ to $f(\lambda)$ at point $(0,0)$ and we solve the equation $l_{\text {mid }}(\lambda)=-d(\lambda)$, then we get

$$
\lambda_{m}^{*}=-\frac{7}{6} \frac{g h \rho u \delta}{u^{2}-g h(1+\rho \delta)} .
$$

Let us consider $\lambda_{1}<\lambda_{2}<\lambda_{3}$ the roots of the polynomial (3.10). We shall use the approximations given by $\lambda_{-}^{*}<\lambda_{m}^{*}<\lambda_{+}^{*}$.

The approximation $\lambda_{+}^{*}$, is an upper bound for $\lambda_{3}$, while $\lambda_{-}^{*}$ is a lower bound of $\lambda_{1}$.

\subsection{Stationary solutions}

In this section we briefly consider some relevant stationary solutions of the system (3.4), when the source term related to de sediment erosion and deposition processes and friction term are neglected. In that case, the momentum conservation equations writes

$$
u\left(\partial_{t}(h \rho)+\partial_{x}(h \rho u)\right)+h \rho\left(\partial_{t} u+u \partial_{x} u\right)+\frac{g}{2} h^{2} \partial_{x} \rho=-g h \rho \partial_{x}(\eta) .
$$


Taking into account the mass conservation equation in (3.4) and that stationary solutions are independent of time, we get

$$
\partial_{x} \frac{u^{2}}{2}+\frac{g h}{2 \rho} \partial_{x} \rho+g \partial_{x} \eta=0 .
$$

Moreover the volume and mass conservation equation in (3.4) results in

$$
\begin{aligned}
\partial_{x}(h u) & =0, \\
\partial_{x}(h \rho u) & =0 .
\end{aligned}
$$

In particular we are interested in those corresponding to $u=0$. In such case, two different families of stationary solutions appear, those corresponding to lake at rest given by

$$
u=0 ; \quad \rho=\text { cte } ; \quad \eta=\text { cte } .
$$

In the case that the density is not a constant function, we get

$$
\frac{g h^{2}}{2} \partial_{x} \rho+g h \rho \partial_{x} \eta=0 .
$$

Moreover, for the case of flat bottom, we get constant pressure steady states given by

$$
u=0 ; \quad \frac{\rho h^{2}}{2}=\text { cte } ; \quad b=\text { cte } .
$$

\subsubsection{Lake at rest solution with suspended sediment}

It will be also interesting to pay special attention to the case where $u=0$ with suspended sediment in the fluid and the erosion and deposition source terms in (3.4) are retained. No stationary solution appears now, but a simple physical solution is found that corresponds to a still water medium where sediment is progressively deposed. Under water at rest supposition $(u=0)$, the 
mathematical model reads

$$
\begin{aligned}
\partial_{t} h & =\frac{\phi_{b}}{1-p}, \\
\partial_{t}(h \rho) & =\frac{\phi_{b} \rho_{b}}{1-p}, \\
\partial_{x}\left(\frac{1}{2} g h^{2} \rho\right) & =-g h \rho \partial_{x} b \\
\partial_{t} b & =-\frac{\phi_{b}}{1-p} .
\end{aligned}
$$

From equations $(3.29)$ and 3.32 we get

$$
\partial_{t}(h+b)=0,
$$

this means that the water free surface does not change through the time.

Developing equation (3.31), the momentum conservation equation takes the form

$$
\partial_{x}(h+b)=-\frac{h}{2 \rho} \partial_{x} \rho .
$$

We remark that if $\rho$ is a constant function, then the free surface of water does not change spatially.

Taking into account equations 3.30 and 3.29 , we get that

$$
\partial_{t} \rho=\frac{\phi_{b}}{h(1-p)}\left(\rho_{b}-\rho\right) .
$$

From definition of $\rho_{b}(2.29)$ and $\rho$ (2.4), equation (3.36) given

$$
\partial_{t} \rho=\frac{\phi_{b}}{h(1-p)}\left(\left(\rho_{s}-\rho_{0}\right)(1-p-c)\right) .
$$

It is expected that the suspended sediment under water at rest must be deposed at the bottom. At the same time, due to this deposit, the thickness of the bottom increases, water thickness decreases. This will modify the density of the water-sediment mixture as follows:

- The density of mixture is a decreasing function through the time if $c<$ $1-p$.

- The density of mixture is an increasing function through the time if $c>$ $1-p$.

- The density of mixture is a constant function if $c=1-p$. 


\section{Numerical scheme}

In this section we describe the first order numerical finite volume solver that we used to approximate the solutions of system (3.4).

The spatial discretization is done by means of a finite volume mesh composed by cells $C_{i}=\left[x_{i-1 / 2}, x_{i+1 / 2}\right], \quad i=1, \cdots, N$. For the sake of simplicity we shall assume that these cells have a constant size $\Delta x$ and denote by $x_{i+1 / 2}=i \Delta x$ the intercells and $x_{i}=\left(i+\frac{1}{2}\right) \Delta x$ the center of the cells, see Figure 4 . Let $\Delta t$ be the time step and $t^{n}=n \Delta t$.

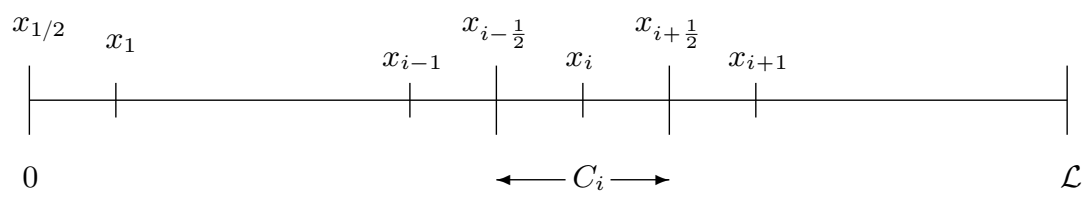

Figure 4: Finite volume mesh.

We use a two-step algorithm to compute the solution of the system (3.4). Incorporating erosion and depositions effects in the second step as it is done in 25, 26, 27, 28,

On the first step, we solve the system

$$
\partial_{t} \mathbf{W}+\mathcal{A}(\mathbf{W}) \partial_{x} \mathbf{W}=\mathbf{0}
$$

using a finite volume well-balanced method and on the second step we solve the ordinary differential equation induced by the erosion deposition and friction source terms

$$
\partial_{t} \mathbf{W}=\mathbf{G}(\mathbf{W})+\mathbf{S}_{\tau}(\mathbf{W})
$$

In order to solve (4.1) we shall use the path-conservative framework. Remark that the system (4.1) contains non conservative products $\mathcal{A}(\mathbf{W}) \mathbf{W}_{x}$, which in general do not make sense within the framework of the theory of distributions. The theory described in [4] allows to give a rigorous definition of weak solutions using a family of Lipschithz continuous paths. This theory has been used to developed the so-called path conservative schemes, see 29 . 
Let $\mathbf{W}_{i}^{n}$ be the approximation of the cell averages of the exact solution at the cell $C_{i}$ at time $t^{n}$

$$
\mathbf{W}_{i}^{n} \cong \frac{1}{\Delta x} \int_{x_{i-1 / 2}}^{x_{i+1 / 2}} \mathbf{W}\left(x, t^{n}\right) d x
$$

One can write a numerical scheme in the form

$$
\mathbf{W}_{i}^{n+1}=\mathbf{W}_{i}^{n+1}-\frac{\Delta t}{\Delta x}\left(\mathbf{D}_{i-\frac{1}{2}}^{+}+\mathbf{D}_{i+\frac{1}{2}}^{-}\right),
$$

with

$$
\begin{aligned}
\mathbf{D}_{i+\frac{1}{2}}^{ \pm}=\mathbf{D}^{ \pm}\left(W_{i}, W_{i+1}\right) & =\frac{1}{2}\left(\mathbf{F}\left(\mathbf{W}_{i+1}^{n}\right)-\mathbf{F}\left(\mathbf{W}_{i}^{n}\right)+\mathbf{B}_{i+\frac{1}{2}}\left(\mathbf{W}_{i+1}^{n}-\mathbf{W}_{i}^{n}\right)\right) \\
& \pm \frac{1}{2} \mathcal{Q}_{i+\frac{1}{2}}\left(\mathbf{W}_{i+1}^{n}-\mathbf{W}_{i}^{n}\right)
\end{aligned}
$$

where the matrix $\mathcal{Q}_{i+\frac{1}{2}}$ represents a viscosity matrix that approximates $\left|\mathcal{A}_{i+\frac{1}{2}}\right|$, the absolute value of the Roe linearization for $\mathcal{A}$ at the intercell. In particular, we will use a PVM method as introduced in [42].

$$
\mathcal{Q}_{i+\frac{1}{2}}=P_{r}^{i+1 / 2}\left(\mathcal{A}_{i+\frac{1}{2}}\right)
$$

where $P_{r}^{i+1 / 2}(x)$ is a polynomial of degree $r$, which approximates the absolute value function.

In this work we employ a parabolic approximation in order to compute the viscosity matrix $\mathcal{Q}_{i+\frac{1}{2}}$. Further details can be found in [43, 44,

$$
P_{2}^{i+1 / 2}\left(\mathcal{A}_{i+\frac{1}{2}}\right)=\alpha_{0} \mathbf{I} d+\alpha_{1} \mathcal{A}_{i+\frac{1}{2}}+\alpha_{2} \mathcal{A}_{i+\frac{1}{2}}^{2} .
$$

where $\alpha_{1}, \alpha_{2}, \alpha_{3}$ are defined in terms of the eigenvalues $\lambda_{1}=\lambda_{\text {min }}, \lambda_{2}=\lambda_{\text {mid }}$, $\lambda_{3}=\lambda_{\max }$ and they are computed by solving the linear system of equations

$$
\left(\begin{array}{ccc}
1 & \lambda_{1} & \lambda_{1}^{2} \\
1 & \lambda_{3} & \lambda_{3}^{2} \\
1 & \lambda_{2} & \lambda_{2}^{2}
\end{array}\right)\left(\begin{array}{c}
\alpha_{0} \\
\alpha_{1} \\
\alpha_{2}
\end{array}\right)=\left(\begin{array}{c}
\left|\lambda_{1}\right| \\
\left|\lambda_{3}\right| \\
\left|\lambda_{2}\right|
\end{array}\right) .
$$


Let us considerer

$$
\begin{aligned}
\gamma_{1} & =-\left(\left|\lambda_{3}\right|\left(\lambda_{1} \lambda_{2}^{2}-\lambda_{1}^{2} \lambda_{2}\right)+\left|\lambda_{2}\right|\left(\lambda_{3} \lambda_{1}^{2}-\lambda_{3}^{2} \lambda_{1}\right)+\left|\lambda_{1}\right|\left(\lambda_{2} \lambda_{3}^{2}-\lambda_{2}^{2} \lambda_{3}\right)\right), \\
\gamma_{2} & =\left|\lambda_{2}\right|\left(\lambda_{1}^{2}-\lambda_{3}^{2}\right)+\left|\lambda_{1}\right|\left(\lambda_{3}^{2}-\lambda_{2}^{2}\right)+\left|\lambda_{3}\right|\left(\lambda_{2}^{2}-\lambda_{1}^{2}\right), \\
\gamma_{2} & =-\left(\left|\lambda_{2}\right|\left(\lambda_{1}-\lambda_{3}\right)+\left|\lambda_{1}\right|\left(\lambda_{3}-\lambda_{2}\right)+\left|\lambda_{3}\right|\left(\lambda_{2}-\lambda_{1}\right)\right), \\
\gamma_{4} & =\left(\lambda_{1}-\lambda_{2}\right)\left(\lambda_{3}\left(\lambda_{3}-\lambda_{1}-\lambda_{2}\right)+\lambda_{1} \lambda_{2}\right),
\end{aligned}
$$

then the solution of the linear system 4.8 is given by

$$
\alpha_{0}=\frac{\gamma_{1}}{\gamma_{4}}, \quad \alpha_{1}=\frac{\gamma_{2}}{\gamma_{4}}, \quad \alpha_{2}=\frac{\gamma_{3}}{\gamma_{4}} .
$$

Remark that here the approximation of the eigenvalues introduced in Section 3.1 shall be used.

In order to ensure the positivity of the numerical scheme, we proceed as follows: if $h_{i+1 / 2}<\varepsilon$, where $\varepsilon$ is threshold value, the viscosity matrix is computed by

$$
P_{2}^{i+1 / 2}\left(\mathcal{A}_{i+\frac{1}{2}}\right)=\alpha_{0} \mathbf{I} d+\alpha_{1} \mathcal{A}_{i+\frac{1}{2}}
$$

where

$$
\alpha_{0}=\frac{\lambda_{3}\left|\lambda_{1}\right|-\lambda_{1}\left|\lambda_{3}\right|}{\lambda_{3}-\lambda_{1}} \quad \text { and } \quad \alpha_{1}=\frac{\left|\lambda_{3}\right|-\left|\lambda_{1}\right|}{\lambda_{3}-\lambda_{1}} .
$$

This means that in a given interface where a wet-dry front is found, the selected PVM at such interface coincides with the HLL scheme. The choice of HLL scheme in such situations will grant the positivity of water and sediment thickness.

\subsection{Numerical treatment of the erosion-deposition source term}

The second step of the method employed is carried out as follow: Let $\mathbf{W}_{i}^{*}$ be the approximation computed using the numerical scheme 4.4. We shall compute $\mathbf{W}_{i}^{n+1}$ in a semi-implicit way as fallows, first the erosion and deposition process are incorporated in the solution

The sediment flux is computed as $\phi_{b, i}^{*}=F_{e, i}^{*}-F_{d, i}^{*}$, being

$$
F_{e, i}^{*}=K_{e r, i}^{*}(1-p), \quad \text { and } \quad F_{d, i}^{*}=K_{d e p, i}^{*} \rho_{i}^{*},
$$


where

$$
K_{e r, i}^{*}=v_{s} E_{s, i}^{*}, \quad \text { and } \quad K_{d e p, i}^{*}=\frac{v_{s}}{\rho_{i}^{*}} c_{i}^{*}\left(0.4\left(\frac{d}{D s g}\right)^{1.64}+1.64\right) .
$$

$$
\begin{aligned}
(h \rho)_{i}^{n+1} & =(h \rho)_{i}^{*}+\frac{\Delta t}{1-p} \rho_{b}\left(K_{e r, i}^{*}(1-p) \frac{b_{i}^{n+1}}{b_{i}^{*}}-\frac{(h \rho)_{i}^{n+1}}{h_{i}^{*}} K_{d e p, i}^{*}\right) 4, \\
(1-p) b_{i}^{n+1} & =(1-p) b_{i}^{*}-\Delta t\left(K_{e r, i}^{*}(1-p) \frac{b_{i}^{n+1}}{b_{i}^{*}}-\frac{(h \rho)_{i}^{n+1}}{h_{i}^{*}} K_{d e p, i}^{*}\right)(.4 .
\end{aligned}
$$

Let us be introduce the following notation

$$
\begin{gathered}
a=\frac{\Delta t \rho_{b}}{1-p} \frac{K_{e r, i}^{*}}{b_{i}^{*}}, \quad e=\frac{\Delta t \rho_{b}}{1-p} \frac{K_{d e p, i}^{*}}{h_{i}^{*}}, \quad c=\frac{\Delta t K_{e r, i}^{*}}{b_{i}^{*}}, \quad d=\frac{\Delta t K_{d e p, i}^{*}}{h_{i}^{*}}, \\
x=(h \rho)_{i}^{n+1} \quad \text { and } \quad y=(1-p) b_{i}^{n+1},
\end{gathered}
$$

and

$$
x_{0}=(h \rho)_{i}^{*} \quad \text { and } \quad y_{0}=(1-p) b_{i}^{*},
$$

then the system of equations 4.13 - 4.14 reduces to

$$
\left(\begin{array}{cc}
1+e & -a \\
-d & 1+c
\end{array}\right)\left(\begin{array}{l}
x \\
y
\end{array}\right)=\left(\begin{array}{l}
x_{0} \\
y_{0}
\end{array}\right)
$$

whose solution is given by:

$$
\begin{aligned}
& x=\frac{(1+c) x_{0}+a y_{0}}{1+c+e}, \\
& y=\frac{d x_{0}+(1+e) y_{0}}{1+c+e} .
\end{aligned}
$$

Remark 4.1. Notice that if $(h \rho)_{i}^{*}$ and $(1-p) b_{i}^{*}$ are positive then $(h \rho)_{i}^{n+1}$ and $(1-p) b_{i}^{n+1}$ will be positive. This is an improvement with respect a explicit approximation of the erosion-deposition terms which does not grant positivity, which would be a major drawback.

From equation 4.13 we get

$$
\frac{\Delta}{1-p} \phi_{b}^{n+1}=\frac{1}{\rho_{b}}\left((h \rho)_{i}^{n+1}-(h \rho)_{i}^{*}\right),
$$

this relation is employed to update the values $h^{n+1}$ and $(h \rho u)^{n+1}$. Finally the friction term is computed in a semi-implicit form. 
Remark 4.2. Remark that the choice of PVM scheme results in the postivity preserving property of the first step of the numerical scheme. Moreover, the semi-implicit approach for the numerical treatment of the erosion and deposition source terms is also positiviy preserving. Therefore this will assure that the global scheme introduced here for the numerical resolution of system (3.4) is positivity preserving.

\section{Extension to bidimensional case}

The mathematical model (3.1) can be easily extended to the bidimensional case. For the sake of completeness we shall describe here the model and numerical scheme in the $2 \mathrm{D}$ case.

The model may be written in compact form as

$$
\begin{aligned}
\partial_{t} \mathbf{W}+\partial_{x} \mathbf{F}_{1}(\mathbf{W})+\partial_{y} \mathbf{F}_{2}(\mathbf{W}) & =\mathbf{B}_{1}(\mathbf{W}) \partial_{x} \mathbf{W}+\mathbf{B}_{2}(\mathbf{W}) \partial_{y} \mathbf{W} \\
& +\mathbf{G}(\mathbf{W})+\mathbf{S}_{\tau}(\mathbf{W}),
\end{aligned}
$$

where $\mathbf{W}=(h, h \rho, h \rho u, h \rho v, b)^{t}$ and

$$
\begin{gathered}
\mathbf{F}_{1}(\mathbf{W})=\left(\begin{array}{c}
h u \\
h \rho u \\
h \rho u^{2}+\frac{1}{2} g h^{2} \rho \\
h \rho u v \\
\frac{1}{1-p} q_{b x}
\end{array}\right) ; \quad \mathbf{F}_{2}(\mathbf{W})=\left(\begin{array}{c}
h v \\
h \rho v \\
h \rho u v \\
h \rho v^{2}+\frac{1}{2} g h^{2} \rho \\
\frac{1}{1-p} q_{b y}
\end{array}\right) \\
\mathbf{B}_{1}(\mathbf{W})=-(g h \rho) e_{3,5}, \quad \mathbf{B}_{2}(\mathbf{W})=-(g h \rho) e_{4,5} \\
\mathbf{G}(\mathbf{W})=\left(\begin{array}{c}
\frac{\phi_{b}}{1-p} \\
\frac{\rho_{b} \phi_{b}}{1-p} \\
\rho \frac{u}{2} \phi_{b} \\
\rho \frac{v}{2} \phi_{b} \\
-\frac{\phi_{b}}{1-p}
\end{array}\right) ; \quad \mathbf{S}_{\tau}(\mathbf{W})=\left(\begin{array}{c}
0 \\
0 \\
\tau_{u} \\
\tau_{v} \\
0
\end{array}\right)
\end{gathered}
$$


where $e_{r, s}$ is the canonical basis of square matrix of order 5 .

The computation of $\phi_{b}$ is made essentially using (2.39) but replacing the absolute value of the velocity $|u|$ by the norm of the vector velocity $|\mathbf{u}|$. The components of friction term $\boldsymbol{\tau}_{f}=\left(\tau_{u}, \tau_{v}\right)$ are computed using 2.47 by taking into account the direction of velocity when the bed shear velocity (2.48) is computed.

The Meyer-Peter \& Müller formulation for the two-dimensional solid transport flux is given by

$$
\mathbf{q}_{\mathbf{b}}=8 Q \operatorname{sgn}(\mathbf{u})\left(\theta-\theta_{c}\right)_{+}^{3 / 2},
$$

where $\operatorname{sgn}(\mathbf{u})$ corresponds to $\mathbf{u} /|\mathbf{u}|$. The Shields parameter, in this case, takes the form:

$$
\theta=\frac{\mu_{f}^{2}}{d\left(\rho_{s}-\rho_{0}\right) h^{1 / 3}}|\mathbf{u}| .
$$

Straightforward computations allow us to prove that the system (5.1) is invariant under ratations (see [45]), that is to say, given a rotation matrix

$$
\mathbf{T}_{\mathbf{n}}=\left(\begin{array}{ccccc}
1 & 0 & 0 & 0 & 0 \\
0 & 1 & 0 & 0 & 0 \\
0 & 0 & n_{x} & n_{y} & 0 \\
0 & 0 & -n_{y} & n_{x} & 0 \\
0 & 0 & 0 & 0 & 1
\end{array}\right)
$$

where $\mathbf{n}=\left(n_{x}, n_{y}\right)^{t}$ is any unitary vector, if we considerer $\mathbf{F}_{\mathbf{n}}=\mathbf{F}_{1}(\mathbf{W}) n_{x}+$ ${ }_{250} \quad \mathbf{F}_{2}(\mathbf{W}) n_{y}$ and $\mathcal{B}(\mathbf{W})=\left(\mathbf{B}_{1}(\mathbf{W}), \mathbf{B}_{2}(\mathbf{W})\right)$ then, the following relations are fulfilled

$$
\begin{aligned}
\mathbf{F}_{\mathbf{n}}(\mathbf{W}) & =\mathbf{T}_{\mathbf{n}}^{-1} \mathbf{F}_{1}\left(\mathbf{T}_{\mathbf{n}} \mathbf{W}\right), \\
\mathcal{B}(\mathbf{W}) \cdot \mathbf{n} & =\mathbf{T}_{\mathbf{n}}^{-1} \mathbf{B}_{1}\left(\mathbf{T}_{\mathbf{n}} \mathbf{W}\right)
\end{aligned}
$$

Following [45, 46] we can see that $\mathbf{T}_{\mathbf{n}} \mathbf{W}$ verifies the system

$$
\partial_{t}\left(\mathbf{T}_{\mathbf{n}} \mathbf{W}\right)+\partial_{\mathbf{n}} \mathbf{F}_{1}\left(\mathbf{T}_{\mathbf{n}} \mathbf{W}\right)=\mathbf{B}_{1}\left(\mathbf{T}_{\mathbf{n}} \mathbf{W}\right) \partial_{\mathbf{n}} \mathbf{W}+\mathbf{Q}_{\mathbf{n}^{\perp}},
$$

where $\mathbf{Q}_{\mathbf{n}^{\perp}}=\mathbf{T}_{\mathbf{n}}\left(-\partial_{\mathbf{n}^{\perp}} \mathbf{F}_{\mathbf{n}^{\perp}}(\mathbf{W})+\left(\mathcal{B}(\mathbf{W}) \cdot \mathbf{n}^{\perp}\right) \partial_{\mathbf{n}^{\perp}} \mathbf{W}\right)$ is the tangential term. This will allow us to easily extend the $1 \mathrm{D}$ numerical scheme to this case. 


\subsection{Numerical solution for the bidimensional model}

255

mesh. In order to solve (5.1), the computational domain is divided into finite volumes $V_{i} \subset \mathbb{R}^{2}$. In this work we use structured meshes made up by rectangular cells whose edges are parallel to the Cartesian axes. Given a finite volume $V_{i}$, $\mathcal{N}_{i}$ is the set of indexes $j$ such that $V_{j}$ is a neighbour of $V_{i} ; E_{i j}$ is the common edge to two neighbour cells $V_{i}$ and $V_{j}$ and $\left|E_{i j}\right|$ represents its length; $\mathbf{n}_{i j}$ is the normal unit vector of the edge $E_{i j}$ pointing towards to the cell $V_{j}$; and $\left|V_{i}\right|$ is the area of the finite volume $V_{i}$.

The path conservative well balanced numerical scheme for the two dimensional model writes

$$
\mathbf{W}_{i}^{n+1}=\mathbf{W}_{i}^{n}-\frac{\Delta t}{\left|V_{i}\right|} \sum_{j \in \mathcal{N}_{i}}\left|E_{i j}\right| \mathcal{D}_{i j}^{-}\left(\mathbf{W}_{i}^{n}, \mathbf{W}_{j}^{n}\right)
$$

where $\mathcal{D}_{i j}^{-}\left(\mathbf{W}_{i}^{n}, \mathbf{W}_{j}^{n}\right)$ is an approximation of the normal flux associated to the edge $E_{i j}$. By using the invariant under rotation property 5.8 we can define

$$
\mathcal{D}_{i j}^{-}\left(\mathbf{W}_{i}^{n}, \mathbf{W}_{j}^{n}\right)=\mathbf{T}_{\mathbf{n}_{i j}}^{-1} D^{-}\left(\mathbf{T}_{\mathbf{n}_{i j}} \mathbf{W}_{i}, \mathbf{T}_{\mathbf{n}_{i j}} \mathbf{W}_{j}\right)
$$

265

\section{Numerical simulations}

In this section, several numerical experiments have been carried out in order to validate the model and the numerical scheme used.

\subsection{Lake at rest}

In this test we try to reproduce numerically the characteristics of lake at 275 
closed flume with flat bottom and we consider that the flume is initially filled with a mixture of water and sediment. The density of sediment is $1.580 \mathrm{~g} / \mathrm{cm}^{3}$, the diameter of the particle is $3.9 \mathrm{~mm}$ and the porosity is 0.47 . The initial concentration of sediment is 0.2 , the initial depth of bottom is $1.05 \mathrm{~m}$ and the initial free surface of water is $3.0 \mathrm{~m}$. The total time simulated is $30 \mathrm{~s}$. The domain $\Omega=[-1,1] \times[-1,1]$ was discretized by using 100 points in the $x$ and $y$ directions, respectively. Free boundary conditions are considered in all boundaries of the domain.
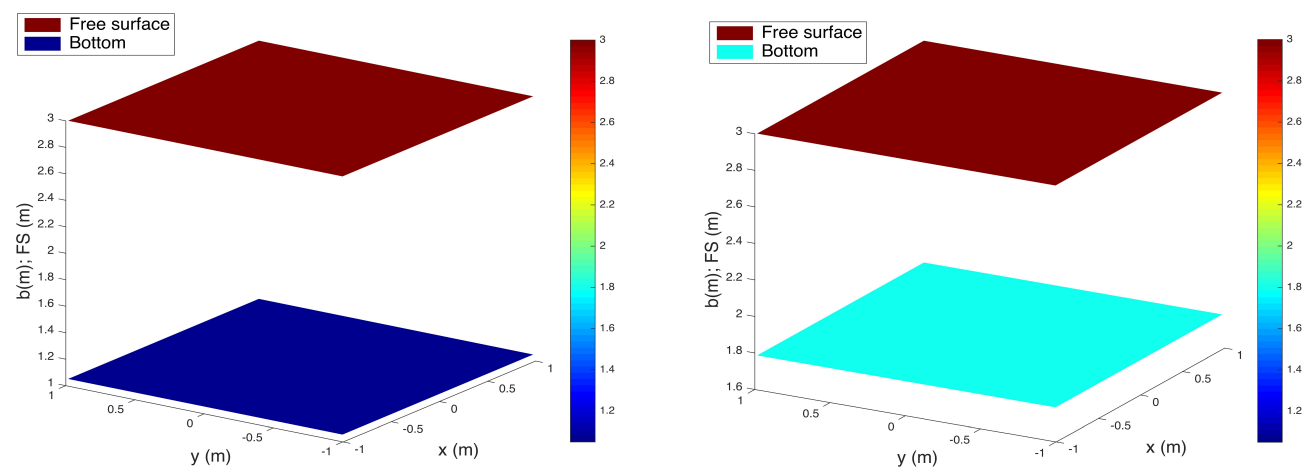

Figure 5: Free surface and bottom at time $t=0 \mathrm{~s}$ (left) and free surface and bottom at $t=30 \mathrm{~s}$ (right).

Figure 5 shows, as expected, that the suspended sediment is deposed at the bottom, therefore the depth of bottom increases. We can notice that the free surface of water remains constant through the time. Remark that the models presented in [35, 47] do not verify this property. In those models, under lake at rest hypothesis, the freshwater mass is increased due to lack of a of a source term in the first equation, which makes that the free surface level does not remains constant through time. Figure 6 shows that the density of water sediment mixture decreases because we have selected a initial value of $c$ such that the relation $c<1-p$ is fulfilled. We can conclude that the numerical scheme 

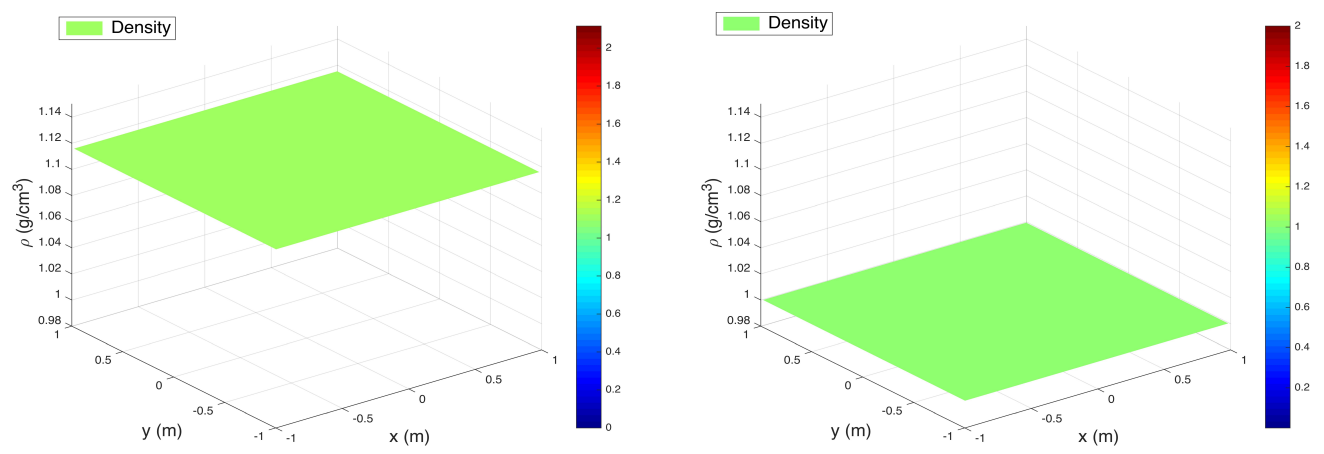

Figure 6: Density at time $t=0 \mathrm{~s}$ (left) and density at $t=30 \mathrm{~s}$ (right).

reproduces correctly the conditions of the lake at rest state for the system (5.1).

\subsection{Dune evolution}

In this test we shall consider bedload transport for the morphological evolution of a dune. We shall consider clear water at initial time and we shall impose an entrance of suspended sediment concentration at left boundary. In this experiment the erosion and deposition terms are neglected. We consider a sedimentary material with the same characteristics described in Section 6.1 and the same domain with the same discretization. The initial conditions are given by

$$
\begin{gathered}
b(\mathbf{x}, 0)= \begin{cases}1.1+0.05 \cos (10|\mathbf{x}|), & \text { if } \quad|\mathbf{x}|<\frac{\pi}{10}, \\
1.05, & \text { Otherwise }\end{cases} \\
h(\mathbf{x}, 0)+b(\mathbf{x}, 0)=2.0, \quad h(\mathbf{x}, 0) u(\mathbf{x}, 0)=1, \\
h(\mathbf{x}, 0) v(\mathbf{x}, 0)=0, \quad \rho(\mathbf{x}, 0)=\rho_{0} .
\end{gathered}
$$

We have employed the Meyer-Peter \& Mülller formulation to compute the bedload discharge (5.5), but in this case we have used

$$
Q=2 d \sqrt{g \frac{\rho_{s}-\rho_{0}}{\rho_{o}}} d
$$


in order to see movement in the bottom at short times. We have imposed the following boundary conditions,

$$
\begin{array}{ll}
h(\mathbf{x}, t) u(\mathbf{x}, t)=1, & \text { for } \quad \mathbf{x} \in[-1,1] \times\{-1,1\}, \quad t>0, \\
h(\mathbf{x}, t) v(\mathbf{x}, t)=0, & \text { for } \quad \mathbf{x} \in\{-1\} \times[-1,1], \quad t>0 .
\end{array}
$$

We shall impose a homogeneous boundary condition on suspended sediment concentration given by

$$
\rho(\mathbf{x}, t)=\rho_{0}+\frac{0.019\left(\rho_{s}-\rho_{0}\right)}{h(\mathbf{x}, t)}, \quad \text { for } \quad \mathbf{x} \in\{-1\} \times[-1,1], t>0 .
$$

295

The remaining boundaries are considered with open boundary condition. The final time is $t=40 \mathrm{~s}$. The numerical results obtained at time $t=40 \mathrm{~s}$ can be seen in Figure 7 .

We can see that the numerical scheme preserves the symmetry, with respect of $x$ axe, of the water free surface, bottom, and discharge in $x$ direction. Remark that the provided numerical solution is smooth. We can see that the bottom at the final time presents a star-shape form which agrees to what is expected (see [48).

\subsection{Simulations of one-dimensional dam breaks flows}

In this section one dimensional experimental dam breaks are simulated in order to do a comparison between the experimental data and the numerical results.

\subsubsection{Experiment 1}

This dam break experiment was performed in the University of Taiwan, Taipe and it was reported in [49. In this experiment the sediment particles considered were artificial spherical pearls covered with a shiny coating, with a diameter of $d=6.1 \mathrm{~mm}$, density $\rho_{s}=1.048 \mathrm{~g} / \mathrm{cm}^{3}$, porosity $p=0.4$, roughness manning coefficient $\mu_{f}=0.03$, critical Shields parameter $\theta_{c}=0.045$ and final time $T=5 t_{0}$, where $t_{0}=\sqrt{h_{0} / g}$ is the hydrodynamic time scale, $h_{0}$ being the initial upstream depth of water. The domain $\Omega=[-0.6,0.6]$ is divided in 500 


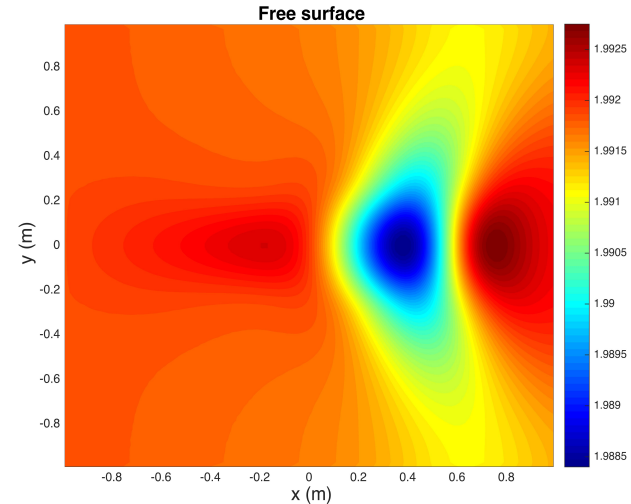

(a)

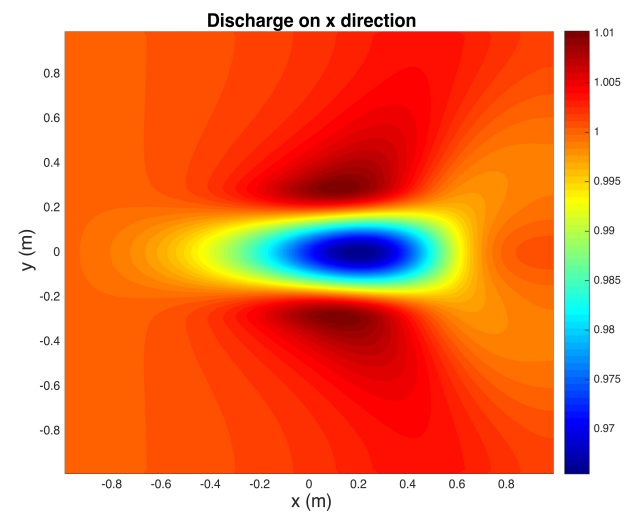

(c)

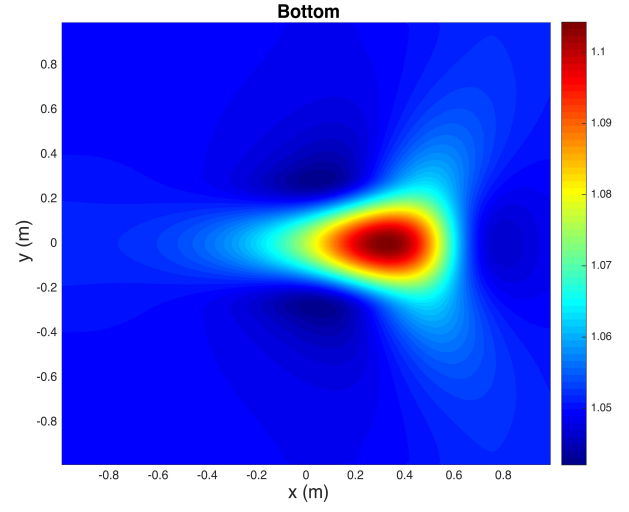

(b)

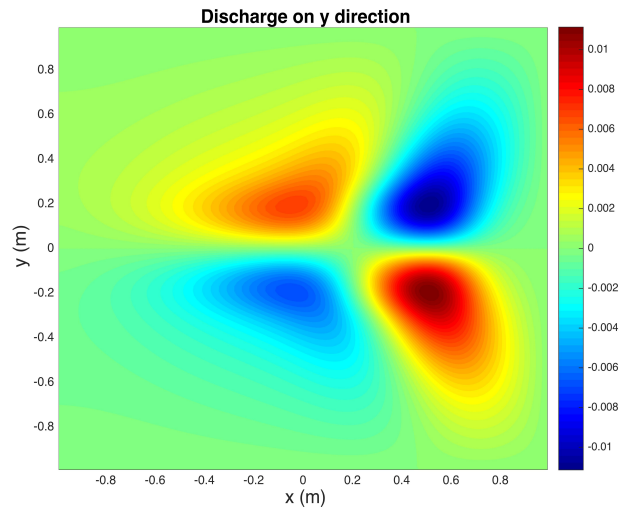

(d)

Figure 7: Numerical results at time $t=40 \mathrm{~s}$; (a) Free surface, (b) Bottom, (c) Discharge on $x$ direction and (d) Discharge on $y$ direction.

points and the initial conditions are given by

$$
h(x, 0)=\left\{\begin{array}{cll}
0.1, & \text { if } & x \leqslant 0, \\
0, & \text { otherwise },
\end{array} u(x, 0)=0, \rho(x, 0)=1, b(x, 0)=0 .\right.
$$

Open boundary conditions are imposed in both ends of the channel. This is done by simply using the ghost cell technique.

A comparison between the numerical results obtained and the experimental data can be seen in Figures 8 to 10 . From these figures we can see that the front of the wave is well captured. We notice that at time $3 t_{0}$ the profile 
of free surface of water is under-estimated while for times $4 t_{0}$ and $5 t_{0}$ the profile computed depicts successfully the shape of free surface provided by the experimental data. On the other hand, for the times $3 t_{0}$ and $4 t_{0}$ the erosion rate computed under-estimate the observed one in the numerical data, while for the time $5 t_{0}$ the erosion rate agrees with the experimental one. As it was pointed out by [49, the non-hydrostatic effects, which are not taken into account by this model, can explain the discrepancies observed between the numerical results and the experimental data. The differences observed in the free surface may be explained by the rapid drop in the hydrostatic pressure. For instance, better approximations of the free surface for this case are achieved in 24] where a nonhydrostatic model is employed. The counterpart being that a non-hydrostatic model is computationally more expensive.

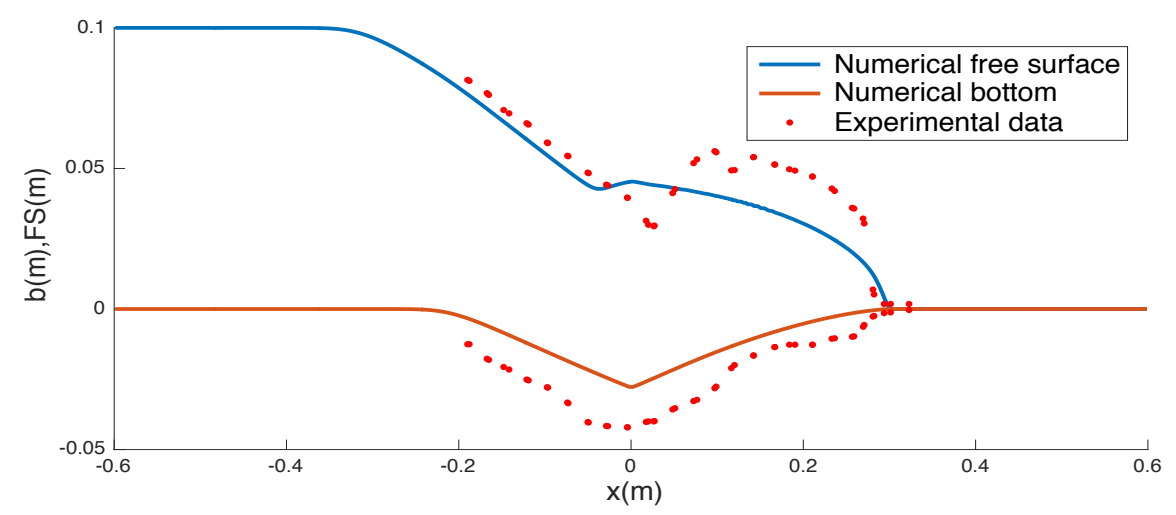

Figure 8: Comparison between numerical results and experimental data at time $t=3 t_{0} s$.

\subsubsection{Experiment 2}

A small scale dam break flux was performed in the Université catholique de Louvain. This experiment was reported in [50]. The sediment considered was composed by cylindrical PVC pellet with diameter $=3.2 \mathrm{~mm}$ and height $=2.8 \mathrm{~mm}$ which is equivalent to a spherical diameter $=3.5 \mathrm{~mm}$, the density ${ }_{330} \rho_{s}=1.540 \mathrm{~g} / \mathrm{cm}^{3}$, the porosity is $p=0.4$, the roughness coefficient $\mu_{f}=0.03$, the critical Shields parameter $\theta_{c}=0.045$ and the total time $T=10 t_{0}$, where 


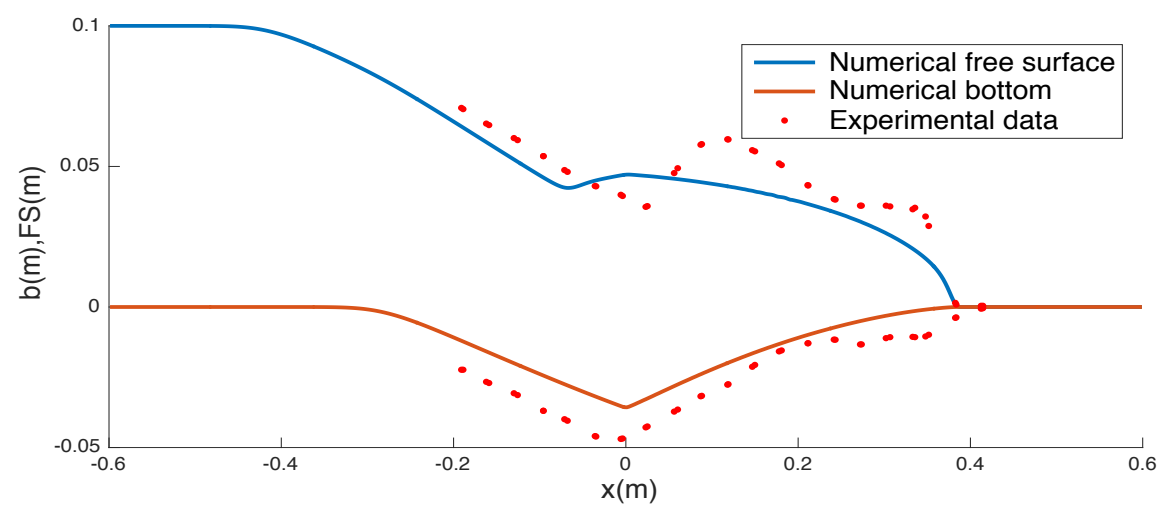

Figure 9: Comparison between numerical results and experimental data at time $t=4 t_{0} \mathrm{~s}$.

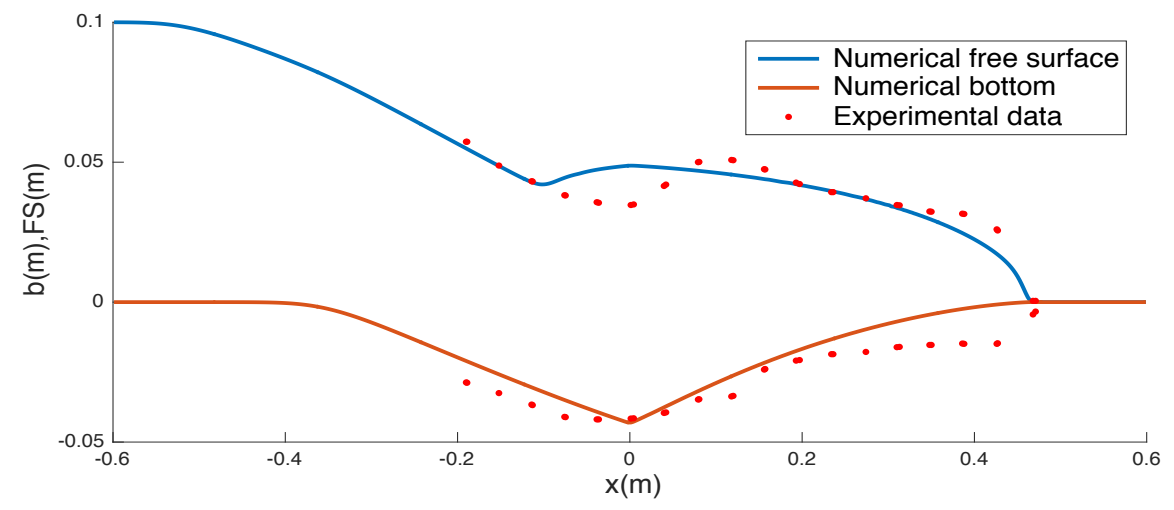

Figure 10: Comparison between numerical results and experimental data at time $t=5 t_{0} s$.

$t_{0}=\sqrt{h_{0} / g}$ is the hydrodynamic time scale, $h_{0}$ being the initial upstream depth of water . The domain $\Omega=[0,2.5]$ is discretized using 500 points and the initial conditions are given by

$$
h(x, 0)=\left\{\begin{array}{rll}
0.1 & \text { if } & x \leqslant 1.25, \\
0 & \text { Otherwise, }
\end{array} u(x, 0)=0, \rho(x, 0)=1, b(x, 0)=0 .\right.
$$

Open boundary conditions are considered at upstream and downstream ends of the channel.

Figures 11 to 13 show that the numerical results capture successfully the advancing front of the water. The time evolution of bottom computed for times 
$t=5 t_{0}$ and $t=7.5 t_{0}$ has a high degree of accuracy. The free surface for these computed times shows a good level of accuracy as well when compared with the reported experimental free surface. For time $t=10 t_{0}$ we can see that the upstream rate of sediment entrainment computed is greater than observed one in the experimental data, while the computed downstream rate of sediment entrainment approximates with a good level of accuracy the experimental data. Again, differences between the shape of the computed free surface and the reported one are associated with the hydrostatic pressure considered in the mathematical model. We remark that the numerical results obtained in this experiment improve the results obtained for instance in [18, 22].

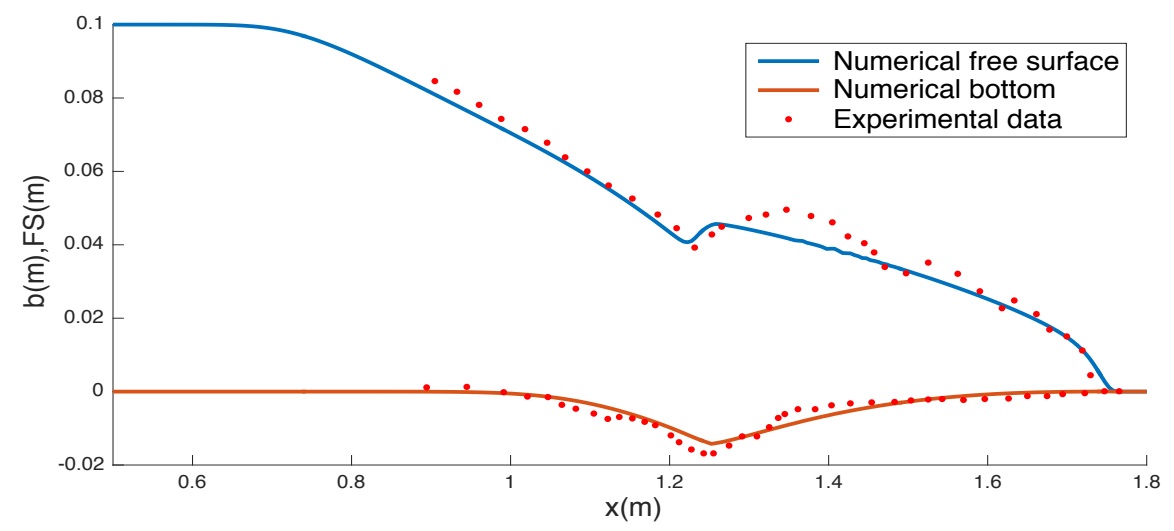

Figure 11: Comparison between numerical results and experimental data at time $t=5 t_{0}$.

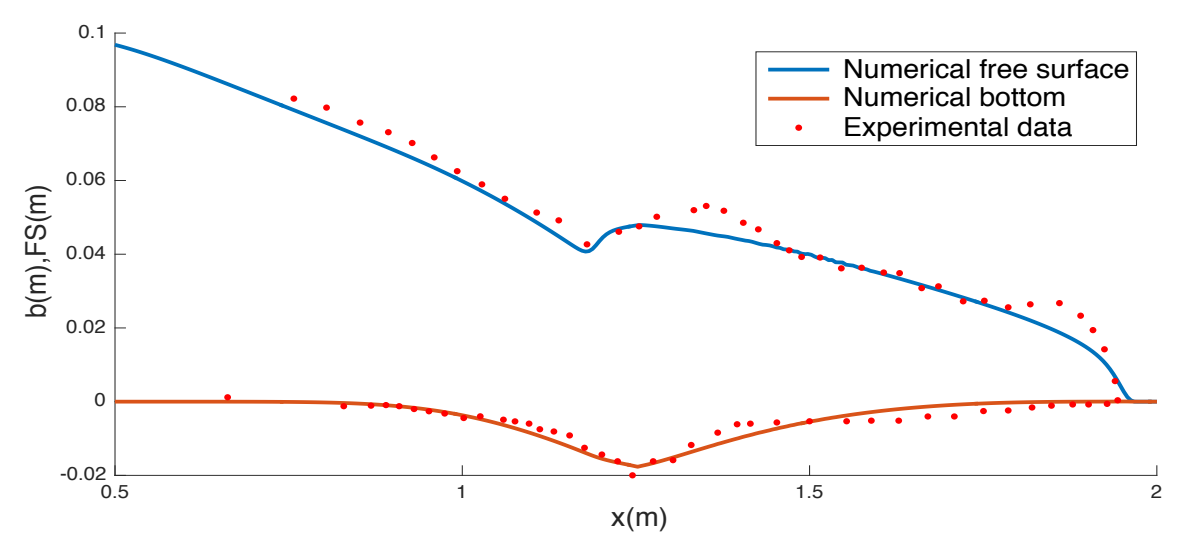

Figure 12: Comparison between numerical results and experimental data at time $t=7.5 t_{0}$. 


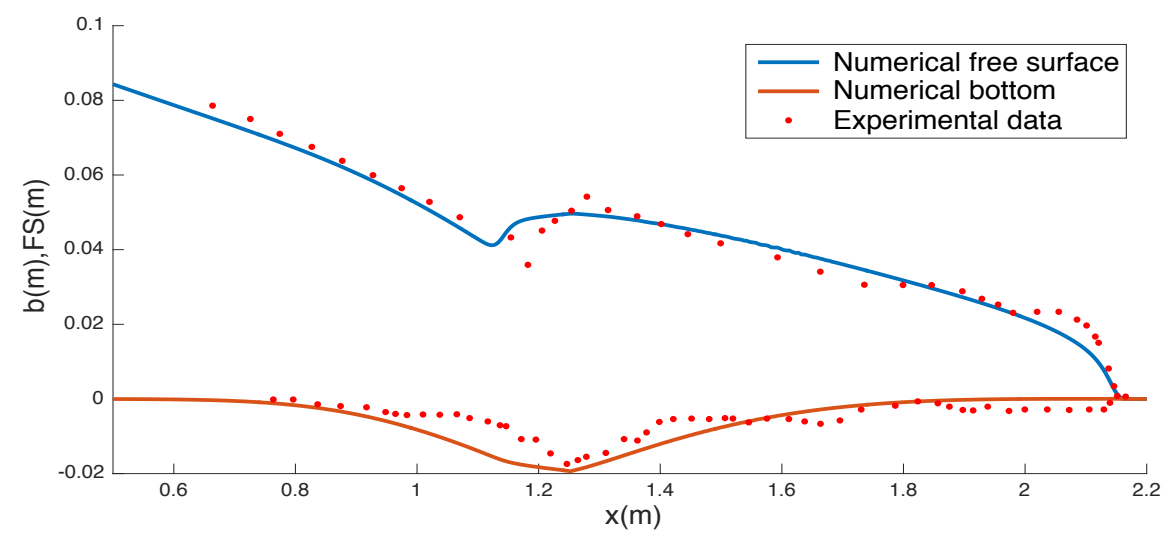

Figure 13: Comparison between numerical results and experimental data at time $t=10 t_{0}$.

\subsubsection{Experiment 3}

This small scale dam break experiment was reported in [51. In this experiment the sediment taken into account is characterised by a diameter of particle $d=3.9 \mathrm{~mm}$, density $\rho_{s}=1.580 \mathrm{~g} / \mathrm{cm}^{3}$, porosity $p=0.47$, the roughness coefficient $\mu_{f}=0.0165$, the critical Shields parameter $\theta_{c}=0.047$ and the total time of compute $T=1.5 \mathrm{~s}$. The domain for this experiment is $\Omega=[-3.0,3.0]$ which has been divided into 500 points. The initial condition are give by

$$
h(x, 0)=\left\{\begin{array}{rll}
0.35 & \text { if } & x \leqslant 0, \\
0 & \text { Otherwise, }
\end{array} u(x, 0)=0, \rho(x, 0)=1, b(x, 0)=0 .\right.
$$

Open boundary conditions are considered at the upstream and downstream ends of the channel.

In Figures 14 to 16 a high level of precision of the numerical results is observed. The shape of computed free surface recreates outstandingly the free surface given by the experimental data at all computed times. The computed shape of bottom approximates successfully the bottom reported by the data, for all computational times. 


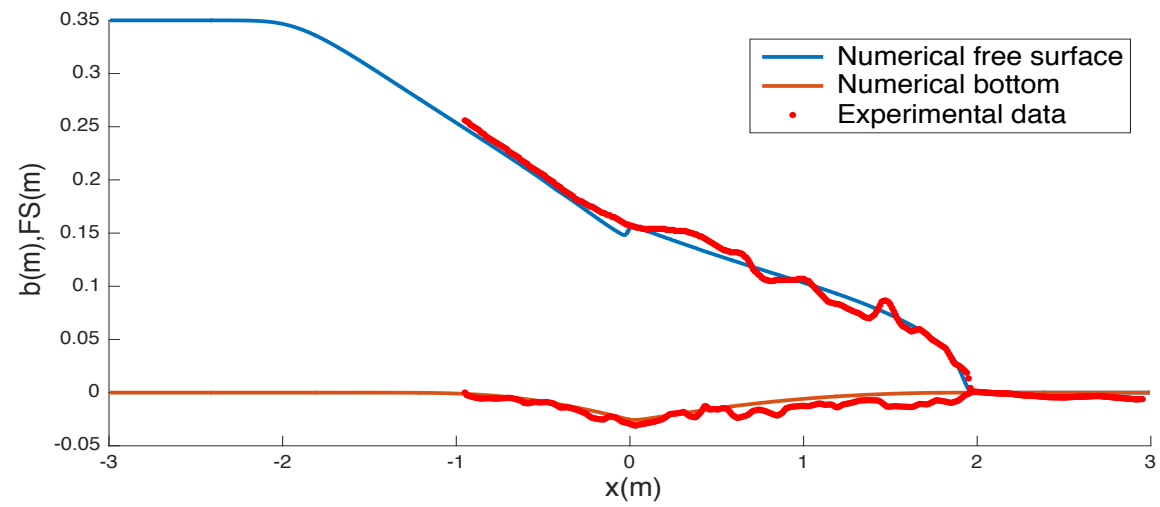

Figure 14: Comparison between numerical results and experimental data at time $t=1.0 \mathrm{~s}$.

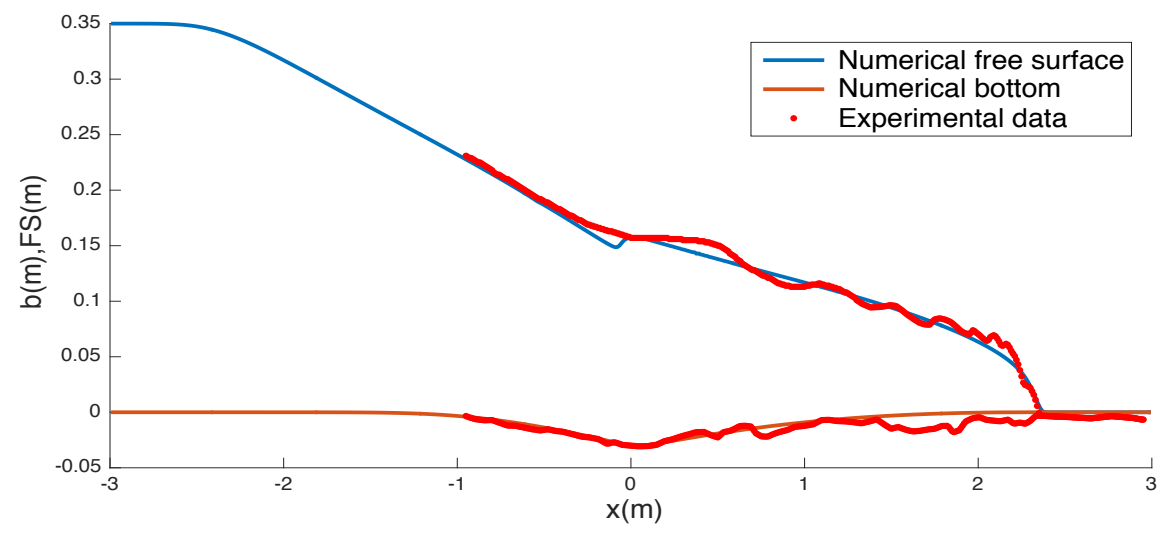

Figure 15: Comparison between numerical results and experimental data at time $t=1.25 \mathrm{~s}$.

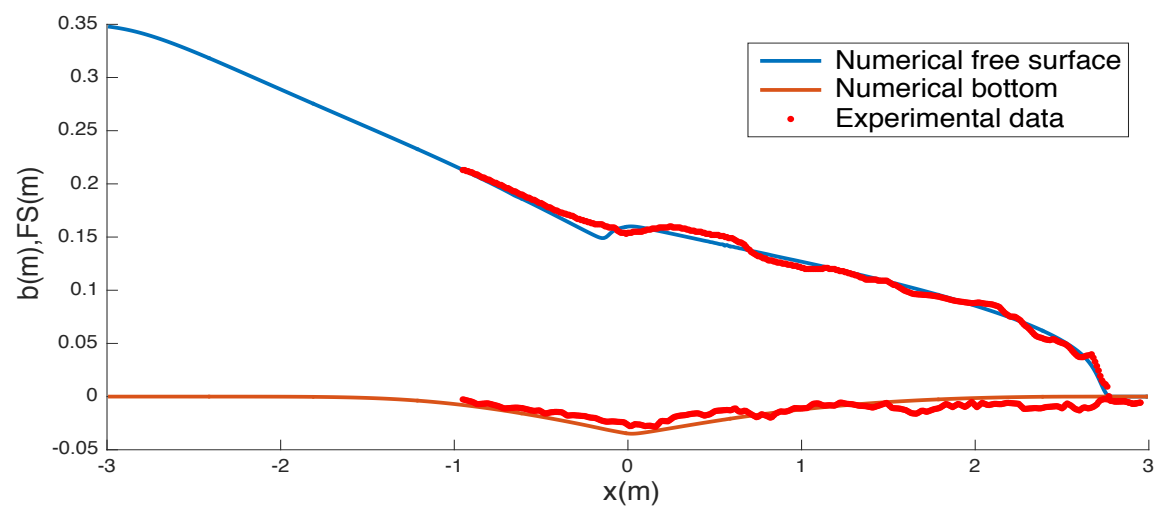

Figure 16: Comparison between numerical results and experimental data at time $t=1.5 \mathrm{~s}$. 


\subsection{Simulations of biimensional dam-breaks flows}

In this section we recreate numerically some bidimensional dam breaks ex-

365

\subsubsection{Experiment 4}

This experiment was carried out at the Hydraulics Laboratory of Université catholique de Louvain and it was reported in [52. The sediment used was coarse, almost uniform sand with a median diameter of $1.72 \mathrm{~mm}$, density $\rho_{s}=$ fully saturated and compacted sand layer of thickness $0.10 \mathrm{~m}$ over whole flume is considered as initial condition for the bottom. The upstream condition for the clear water layer is $0.25 \mathrm{~m}$ and $0 \mathrm{~m}$ for the downstream. In Figure 17 we can see a sketch of the shape of the domain and the considered initial conditions. A free boundary condition is considered at right end of the channel while a wall boundary condition is assumed at remaining boundaries.

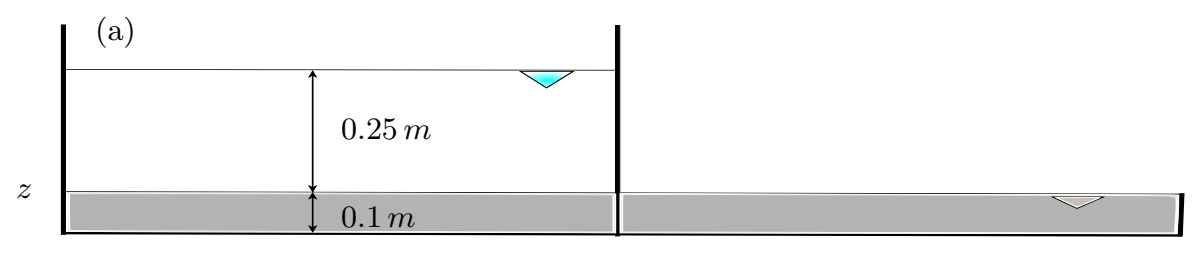

$x$

(b)

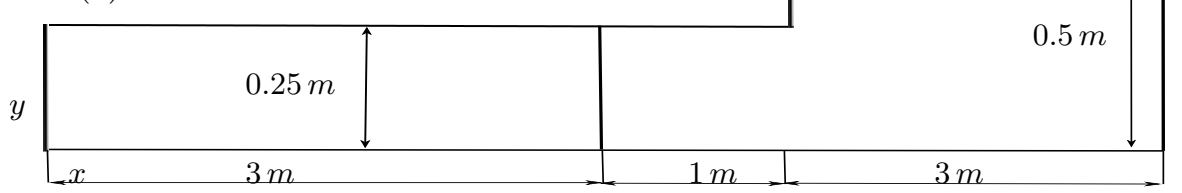

Figure 17: Experimental set-up and initial conditions: (a) Longitudinal profile and (b)plan view.

Figure 18 shows the free surface of the water and the depth of bottom at final simulation time $t=12 \mathrm{~s}$. In Figures 19 to 21 we can see a comparison between the numerical results and the experimental data for the depth of bot- 
tom at different cross sections. From Figure 19 we can see that the numerical results have the same shape than experimental data but with a difference of around $0.01 \mathrm{~m}$. Figure 20 shows that the numerical results under-estimate the experimental data for $y=0.25 \mathrm{~m}$, after that point the numerical results recreate good enough the tendency of the experimental data. The comparison at cross section $x=1.6 \mathrm{~m}$ is showed in Figure 21, we can see that the numerical results adjust successfully the experimental data. Therefore we can conclude that the morphological evolution for this experiment is computed successfully.
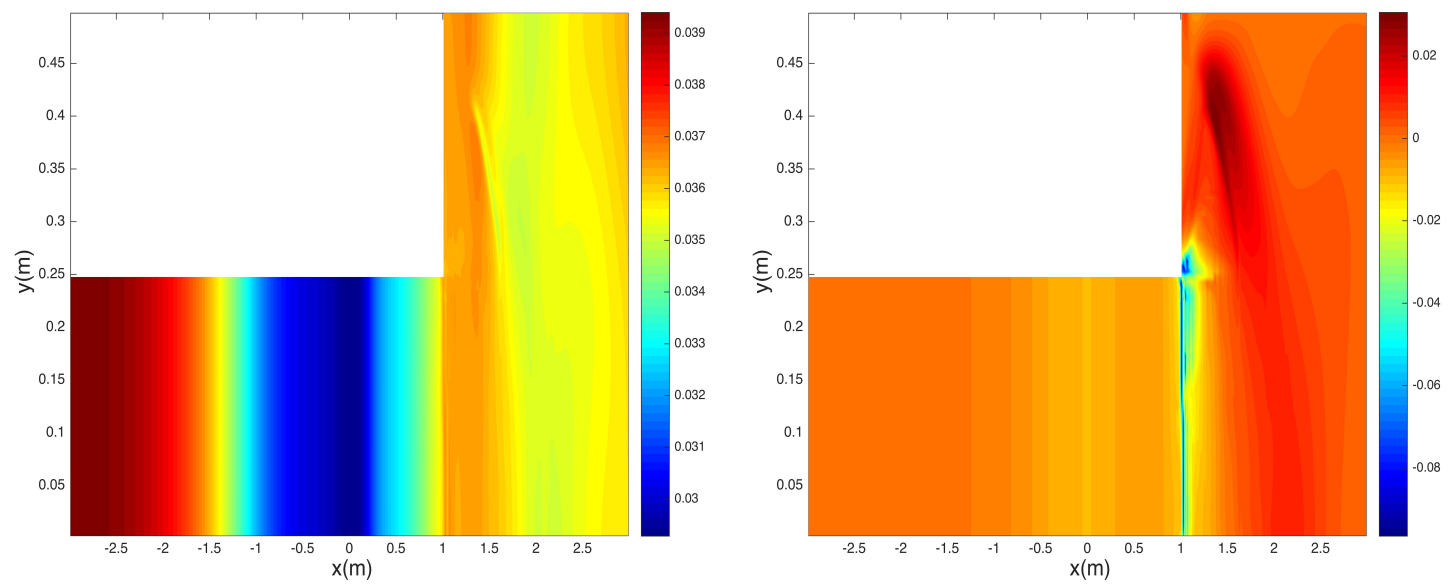

Figure 18: Free surface at $t=12 \mathrm{~s}$ (left) and bottom at $t=12 \mathrm{~s}$ (right).

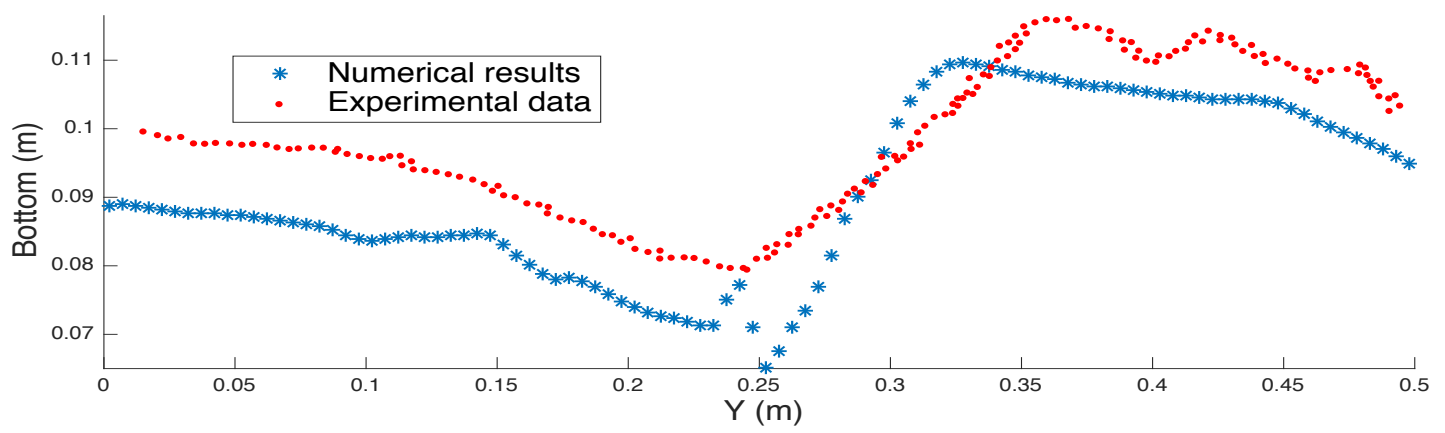

Figure 19: Cross section at $x=1.15 \mathrm{~m}$ at time $t=12 \mathrm{~s}$. 


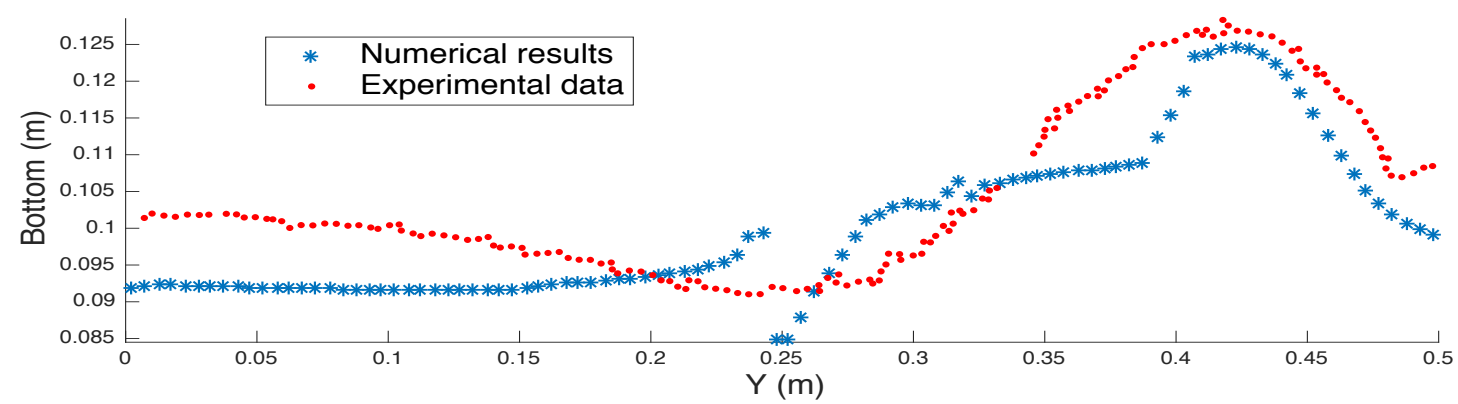

Figure 20: Cross section at $x=1.3 \mathrm{~m}$ at time $t=12 \mathrm{~s}$.

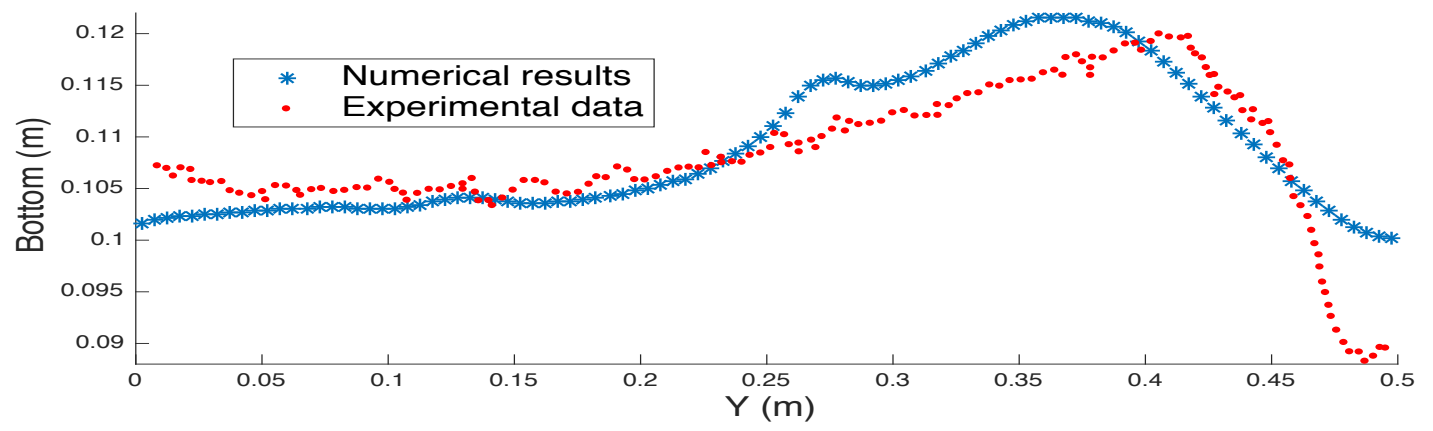

Figure 21: Cross section at $x=1.6 \mathrm{~m}$ at time $t=12 \mathrm{~s}$.

Figure 22 shows the comparison of temporal evolution of free surface of water, we can see that the the evolution computed by the numerical results follows the shape of the temporal evolution given by the experimental data, little difference can be observed after the eighth second. In Figure 23 we can notice that before $t=3 \mathrm{~s}$ the numerical results depict correctly the time evolution of the experimental data, between $t=3 \mathrm{~s}$ and $t=5 \mathrm{~s}$ the numerical results under-estimate the values reported by the data, after $t=6 \mathrm{~s}$ we can see an over-estimation gives by the numerical results. It can be observed that the approach presented here improves the numerical results obtained in 53 for the bed evolution and they are comparable to the ones obtained in [22]. 


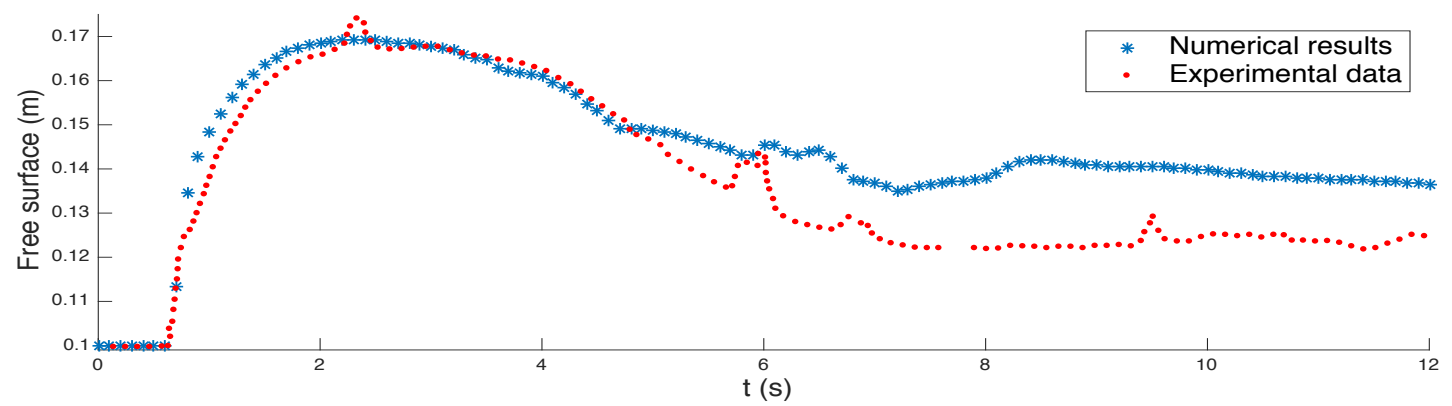

Figure 22: Temporal evolution of free surface at point $(4.2,0.125)$.

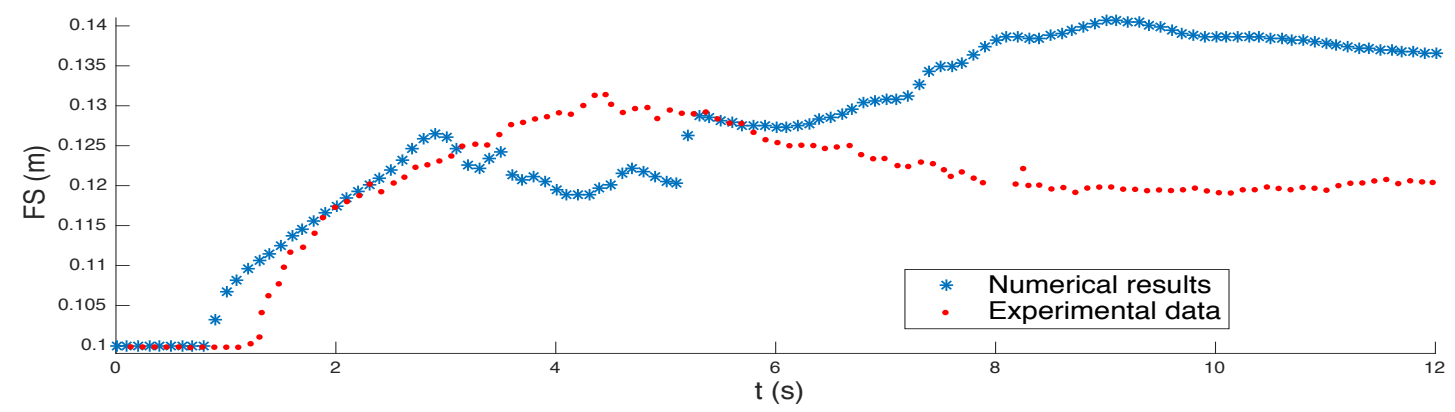

Figure 23: Temporal evolution of free surface at point $(4.2,0.375)$.

\subsubsection{Experiment 5}

This experiment has been conducted at the Hydraulics Unit of Mechanical and Civil Engineering Laboratory, Université de Louvain. The set-up of the flume and its dimensions can be seen in Figure 24. This experiment was reported in [54. The sediment used was coarse sand characterized by a diameter $d=$ $1.61 \mathrm{~mm}$ with density $\rho_{s}=2.63 \mathrm{~g} / \mathrm{cm}^{3}$ and porosity $p=0.42$, the manning roughness coefficient reported was $\mu_{f}=0.0165 \mathrm{~s} / \mathrm{m}^{1 / 3}$ for the movable bottom 405 and $\mu_{f}=0.010 \mathrm{~s} / \mathrm{m}^{1 / 3}$ for the fixed bed. The boundary conditions consisted of a closed wall at the upstream of the flume and free boundary condition at the downstream of the flume. The remaining boundary conditions correspond to wall condition. The initial water level in the upstream is denoted by $\eta_{0}$, while the initial water level in the interval $0<x<9$ is defined by $\eta_{1}$, and initial 
water level at interval $x>9$ is denoted by $\eta_{2}$. The initial thickness of sand layer is $h_{s}=0.085 \mathrm{~m}$, these initial conditions can be seen in Table 1. During the experiment test repeatability was cheked to measure its level of repeatability, further details can be found in [54].
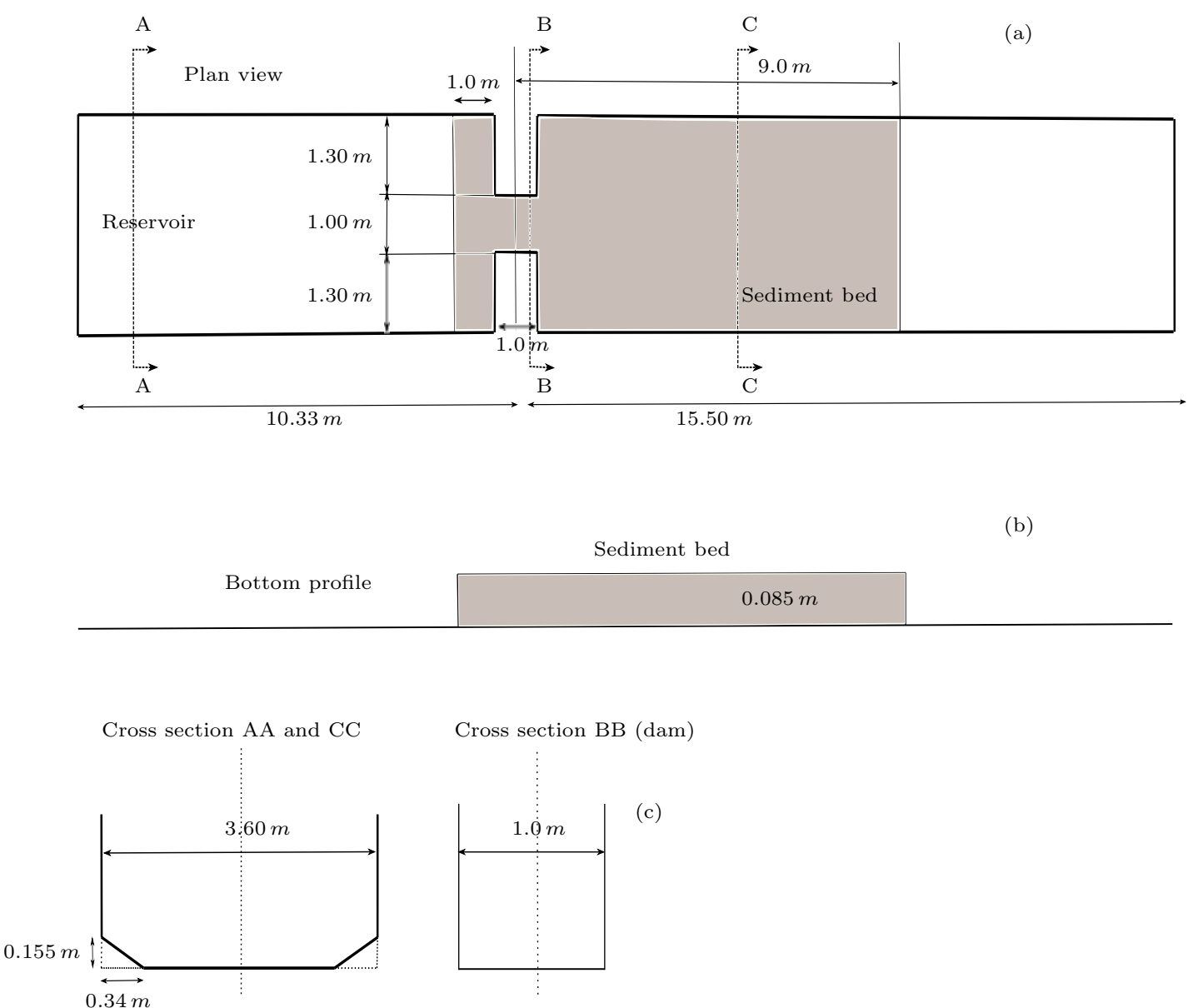

Figure 24: Experimental set-up, flume dimensions: (a) plan view, (b) elevation and (c) crosssections.

Figure 25 shows the final state for the free surface of water and the depth of bottom after $20 s$ of simulation. In Figures 26 to 28 we can see the comparison 
Table 1: Initial conditions for the level of water.

\begin{tabular}{cccc}
\hline$\eta_{0}(m)$ & $\eta_{1}(m)$ & $\eta_{2}(m)$ & $h_{s}(m)$ \\
\hline 0.4710 & 0.085 & 0.0 & 0.085 \\
\hline
\end{tabular}
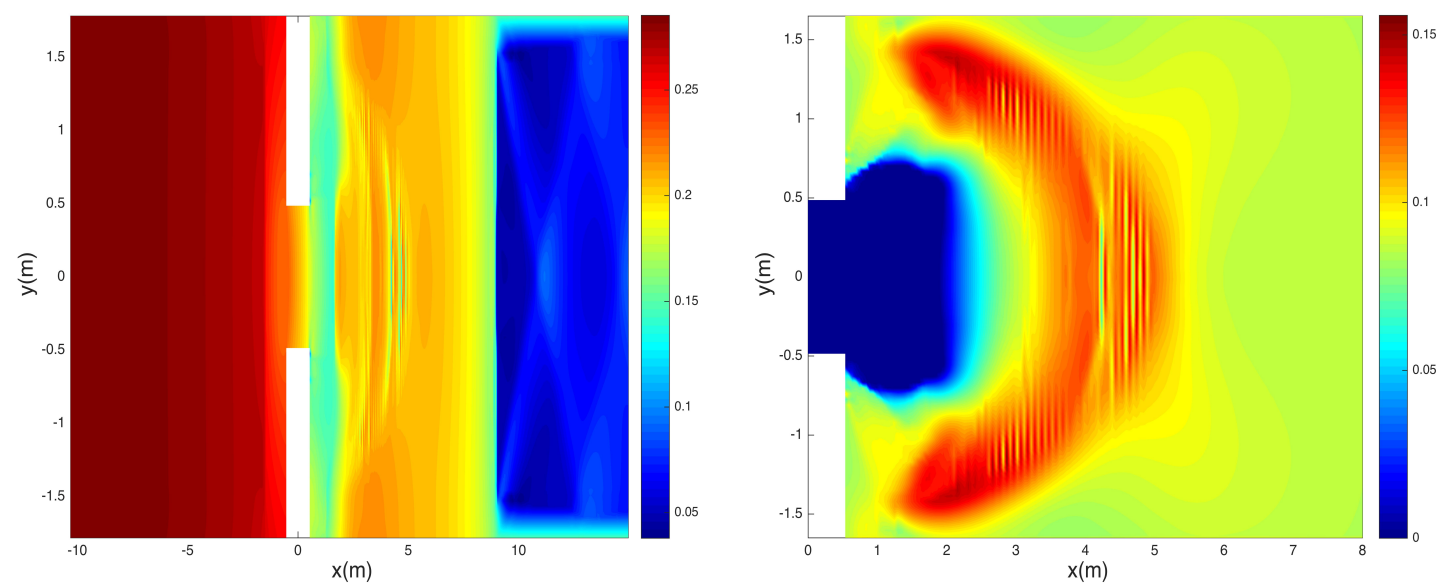

Figure 25: Free surface of water at $t=20 \mathrm{~s}$ (left) and bottom at $t=20 \mathrm{~s}$ (right).

between the numerical results and the experimental data for the final bottom at different longitudinal sections. In Figure 26 we can see that the morphological evolution is well recreated by the numerical results. Notice that in the interval $0<x<2$ the rate of erosion in the numerical results is greater than the observed one in the experimental data, but at the remaining comparison section the numerical results capture successfully the shape of experimental data. Figure 27 shows that the general shape of the experimental data is recovered by the numerical results, little differences can be observed at $1<x<2$, but the approximations computed have a good level of accuracy. In Figure 28 we can observe that the numerical results recreate successfully the experimental data. The deposition rate is well computed in the section $0<x<2$, after this interval the deposition rate is under-estimated (or the erosion rate is over-estimated).

During the experiment, the temporal evolution of water level was measured and the gauge positions are stated in Table 2. In Figures 29 to 32 we can see 


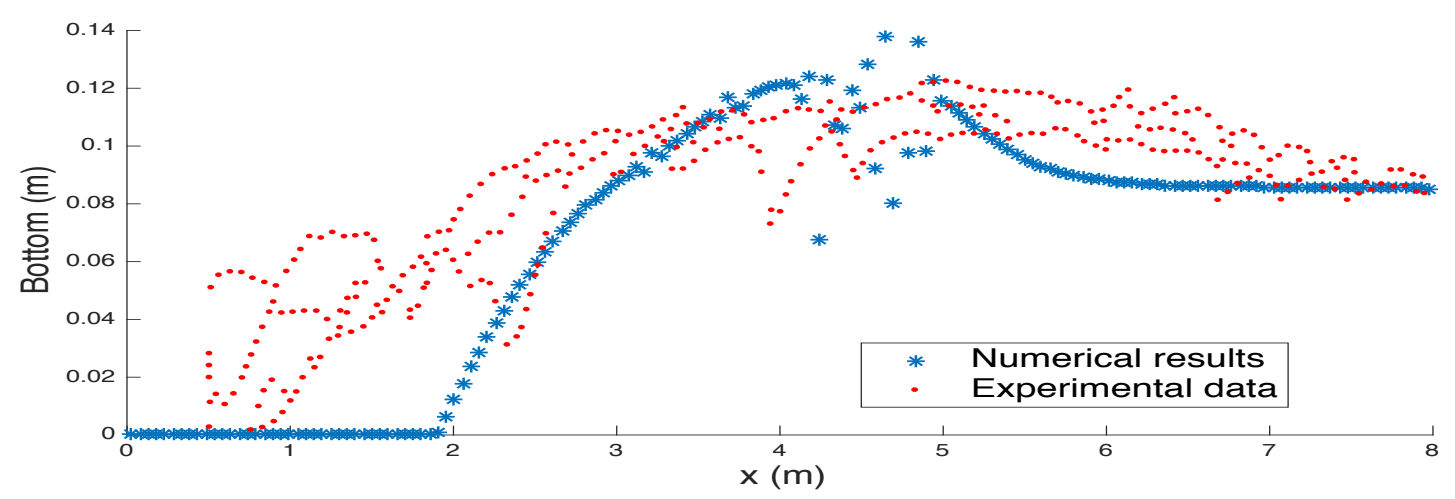

Figure 26: Bottom surface at section $y=0.2$ at time $t=20 \mathrm{~s}$.

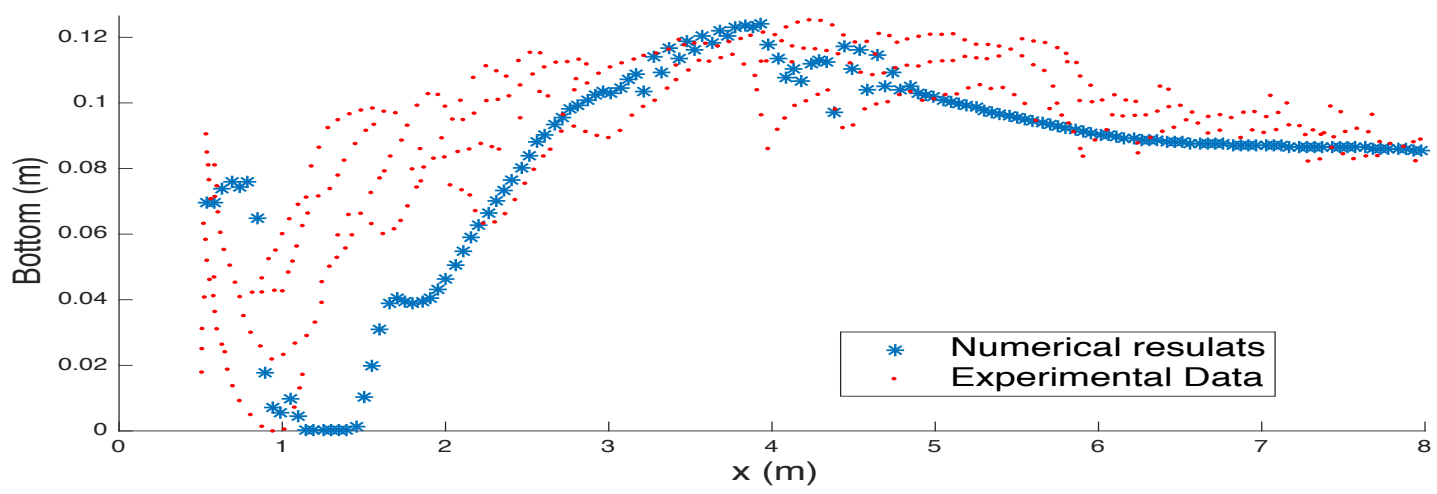

Figure 27: Bottom surface at section $y=0.6$ at time $t=20 \mathrm{~s}$.

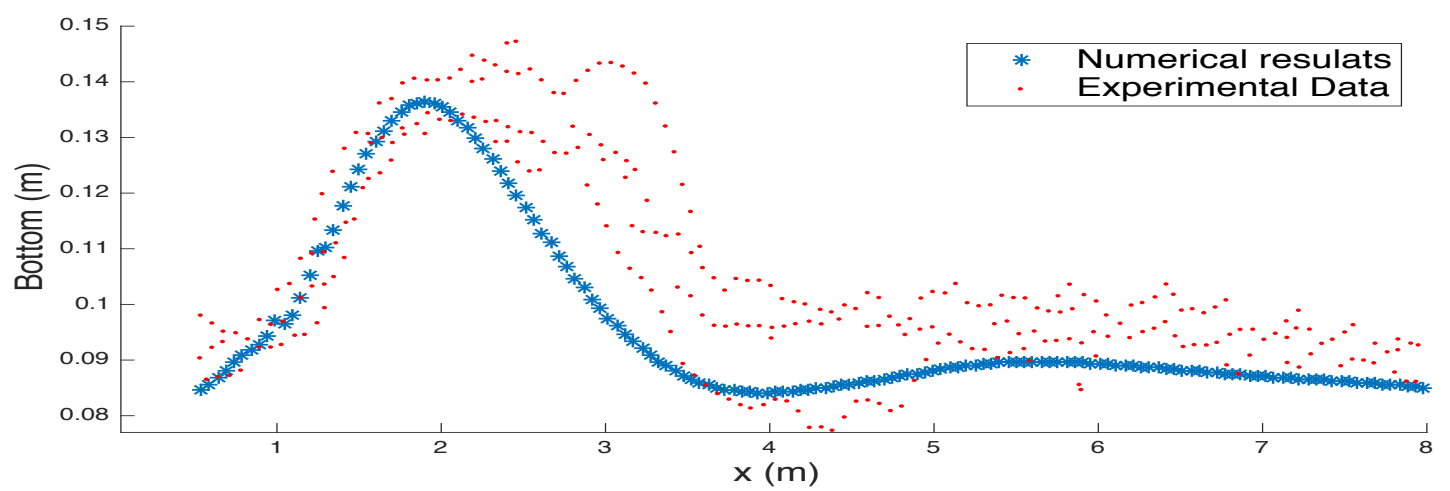

Figure 28: Bottom surface at section $y=1.45$ at time $t=20 \mathrm{~s}$. 
Table 2: Gauge locations.

\begin{tabular}{ccc}
\hline \hline Gauge Id & $x(m)$ & $y(m)$ \\
\hline US1 & 0.640 & -0.500 \\
US3 & 0.640 & 0.165 \\
US5 & 1.940 & -0.990 \\
US6 & 1.940 & -0.330 \\
\hline \hline
\end{tabular}

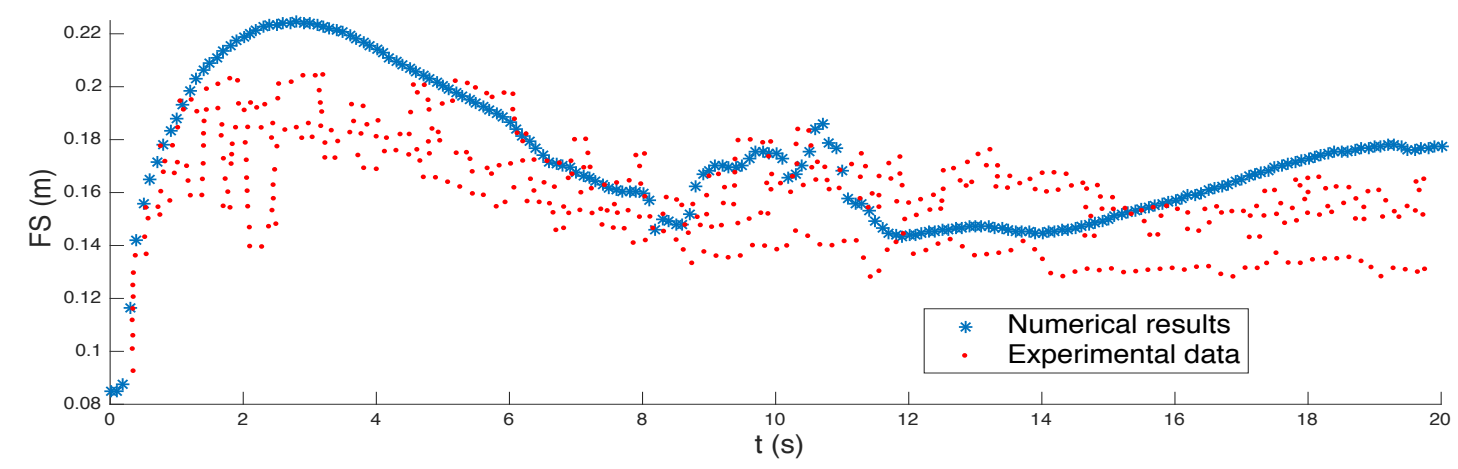

Figure 29: Temporal evolution of free surface at point US1. 


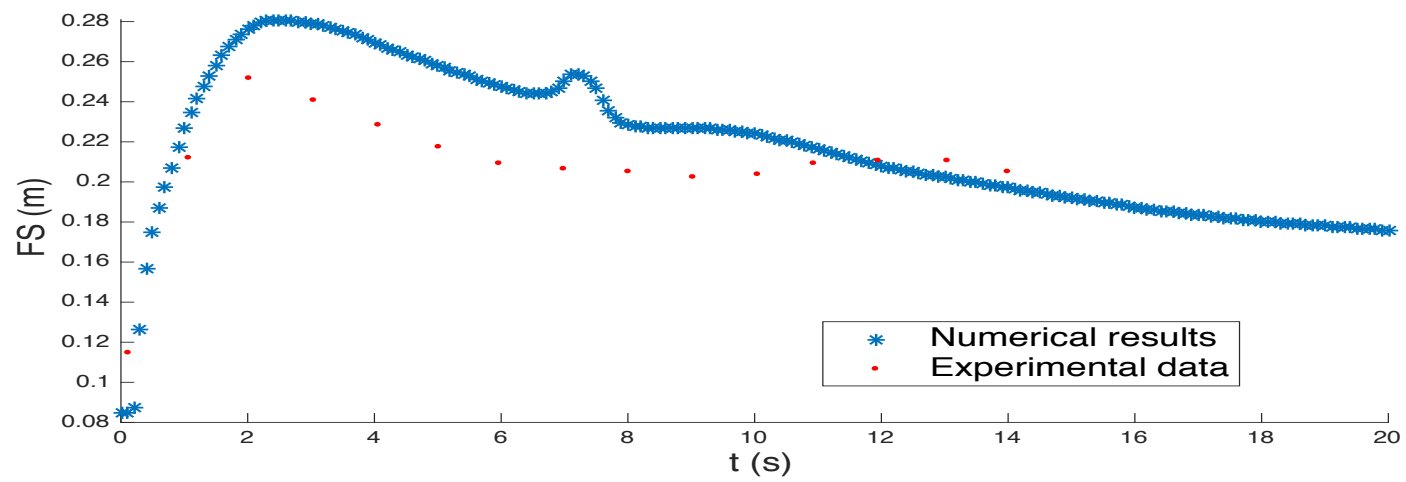

Figure 30: Temporal evolution of free surface at point US3.

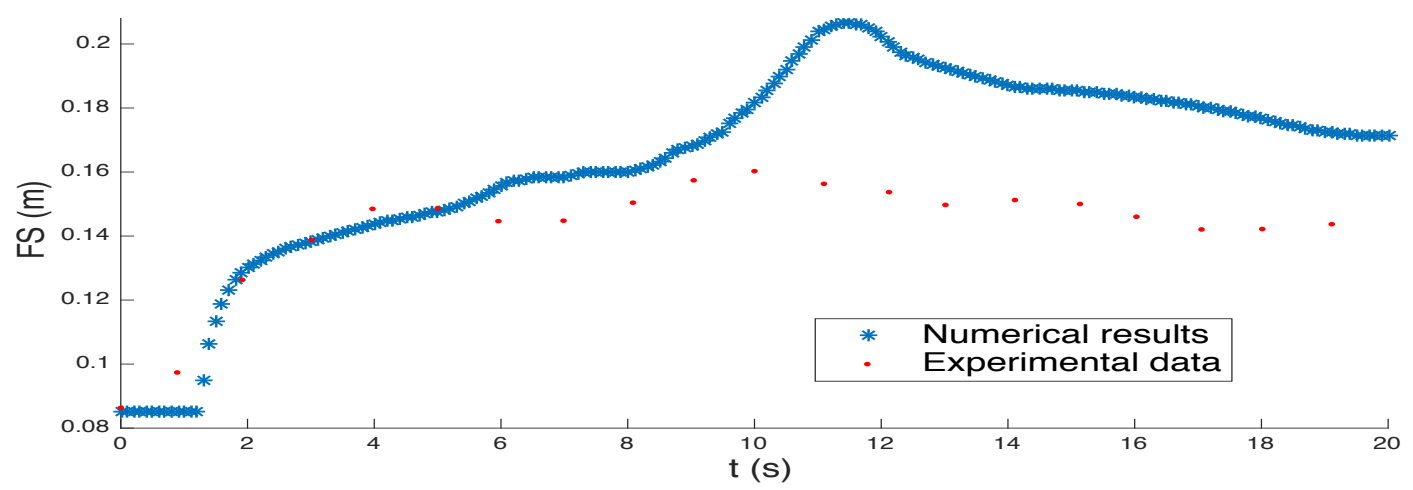

Figure 31: Temporal evolution of free surface at point US5.

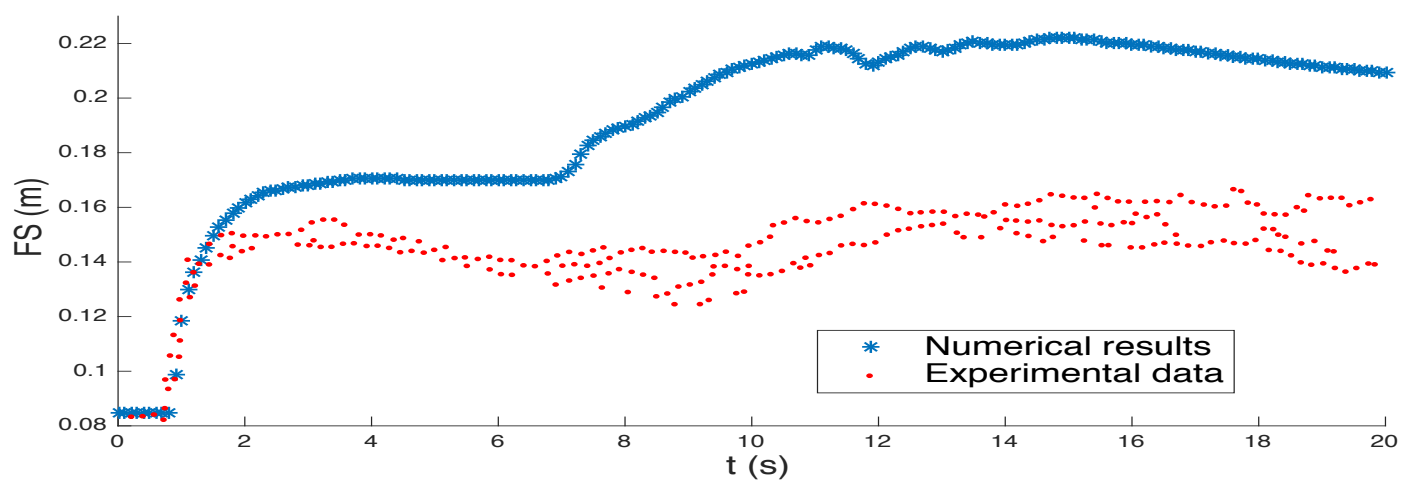

Figure 32: Temporal evolution of free surface at point US6. 


\section{Conclusions}

We have deduced a new model for suspended and bedload sediment transport under the shallow water hypothesis. The model does not make a Boussinesq type hypothesis. The numerical scheme proposed is based on a two-step algorithm. First the underlying hyperbolic system is solved using a PVM path conservative scheme, which is a fast and robust scheme. Then the source terms corresponding to erosion and deposition are applied. This is done by using a semi-implicit approach which allow us to guarantee that the computed densities remain positive, so that it results in a robust way of treating such terms.

From the comparison done between the numerical results and the experimental data we can conclude that the mathematical model and the numerical scheme proposed in this work provided successful results. Notice from the one dimensional experiments that a high level of accuracy in the results is obtained. The model captures correctly the advance of the front wave and the shape the of free surface. Regarding to the morphological evolution, a high level of accuracy is observed as well. Some differences may be observed, specially for small times, between the numerical and experimental results. These discrepancies may be explained by the presence of non hydrostatic effects which are not considered in the model. Doing so would make it more cumbersome and less efficient.

For the two dimensional experiments, on the one hand we can conclude that the morphological evolution is computed successfully, the rate of erosion and deposition are according with the observed ones in the experimental data, but the amplitudes were underestimated. On the other hand, the free surface of water was good enough computed in some gauge while for other a overestimation of that is obtained.

In general, the mathematical model and the numerical scheme proposed here is able to recreate transient flows over movable bottom in an accurate and efficient way. 


\section{Acknowledgement}

This research has been partially supported by the research project Modelling and computation of shocks and interfaces with reference 8.06.UE/47.8007 as well by the Spanish Government and FEDER through the coordinated Research projects MTM 2015-70490-C2-(1/2)-R and RTI2018-096064-B-C2(1/2).

\section{References}

[1] D. Subhasish, Fluvial Hydrodynamics, Hydrodynamic and Sediment Transport Phenomena, GeoPlanet: Earth and Planetary Sciences, SpringerVerlag Berlin Heidelberg, 2014.

[2] F. M. Exner, Über die wechselwirkung zwischen wasser und geschiebe in flüsen, Akad. Wiss. Wien Math. Naturwiss, Klasse 134 (1925) 165-2014.

[3] E. Meyer-Peter, R. Müller, Formulas for bed-load transport, Tech. rep., 2nd Meet. Int. Assoc. Hydraul. Struct. Res., Stockholm, 39-64 (1948).

n [4] H. A. Einstein, The bed load function for sediment transportation in open channel flows, Bulletin 1026, Department of Agriculture, Soil Conservation Service, Washington, D.C. (1950).

URL http://purl.umn .edu/156389

[5] A. J. Grass, Sediment transport by waves and currents, Tech. Rep. FL29, SERC. London Cent. Mar (1981).

[6] L. C. Van Rijn, Sediment transport, part I: Bed load transport, Journal of Hydraulic Engineering 110 (10) (1984) 1431-1456. URL http://ascelibrary.org/doi/abs/10.1061/\%28ASCE\% 290733-9429\%281984\%29110\%3A10\%281431\%29

¿ [7] V. Caleffi, A. Valiani, A. Bernini, High-order balanced cweno scheme for movable bed shallow water equations, Advances in Water Resources 30 (4) 495 ㄴ (2007) 730 - 741. doi:https://doi.org/10.1016/j.advwatres.2006. 
06.003

URL http://www.sciencedirect.com/science/article/pii/ S0309170806001072

[ [8] M. J. Castro-Díaz, E. D. Fernández-Nieto, A. M. Ferreiro, Sediment transport models in shallow water equations and numerical approach by high order finite volume methods, Computers \& Fluids 37 (3) (2008) 299 - 316. URL https://doi.org/10.1016/j.compfluid.2007.07.017

q [9] X. Liu, B. Landry, M. García, Two-dimensional scour simulations based

1 0 coupled model of shallow water equations and sediment transport on unstructured meshes, Coastal Engineering 55 (10) (2008) 800 - 810. doi:https://doi.org/10.1016/j.coastaleng.2008.02.012.

10 URL http://www.sciencedirect.com/science/article/pii/ S0378383908000525

[10] A. Canestrelli, A. Siviglia, M. Dumbser, E. F. Toro, Well-balanced 510 high-order centred schemes for non-conservative hyperbolic sysa tems. applications to shallow water equations with fixed and mobile bed, Advances in Water Resources 32 (6) (2009) 834 - 844 . doi:https://doi.org/10.1016/j.advwatres.2009.02.006.

1 URL http://www.sciencedirect.com/science/article/pii/ $515 \quad$ S0309170809000256

[11] J. Murillo, P. García-Navarro, An exner-based coupled model for twodimensional transient flow over erodible bed, Journal of Computational Physics 229 (23) (2010) $8704-8732$.

URL https://doi.org/10.1016/j.jcp.2010.08.006

520 [12] S. Soares-Frazão, Y. Zech, Hllc scheme with novel wave-speed estimators appropriate for two-dimensional shallow-water flow on erodible bed, International Journal for Numerical Methods in Fluids 66 (8) (2011) 1019-1036. doi:10.1002/fld.2300.

URL https://onlinelibrary.wiley.com/doi/abs/10.1002/fld.2300 
[13] C. Juez, J. Murillo, P. García-Navarro, A 2D weakly-coupled and efficient numerical model for transient shallow flow and movable bed, Advances in Water Resources 71 (2014) 93 - 109.

URL https://doi.org/10.1016/j.advwatres.2014.05.014

[14] H. M. Pantin, Interaction between velocity and effective density 530 in turbidity flow: Phase-plane analysis, with criteria for auto-

1 Suspension, Marine Geology 31 (1) (1979) 59 - 99. doi:https: //doi.org/10.1016/0025-3227(79)90057-4.

a URL http://www.sciencedirect.com/science/article/pii/ 0025322779900574

535 [15] Z. Cao, G. Pender, S. Wallis, P. Carlling, Computational dam-break hydraulics over erodible sediment bed, Journal of Hydraulic Engineering 130 (7) (2004) 689-703.

n URL http://ascelibrary.org/doi/abs/10.1061/\%28ASCE\% $290733-9429 \% 282004 \% 29130 \% 3 A 7 \% 28689 \% 29$

540 [16] P. Hu, Z. Cao, Fully coupled mathematical modeling of turbidity currents over erodible bed, Advances in Water Resources 32 (1) (2009) 1 - 15. URL https://doi.org/10.1016/j.advwatres.2008.07.018

[17] G. Simpson, S. Castelltort, Coupled model of surface water flow, sediment transport and morphological evolution, Comput545 a ers \& Geosciences 32 (10) (2006) 1600 - 1614. doi:https: //doi.org/10.1016/j.cageo.2006.02.020

1. URL http://www.sciencedirect.com/science/article/pii/ S0098300406000458

[18] W. Wu, S. S. Wang, One-dimensional modeling of dam-break flow over 550 movable beds, Journal of Hydraulic Engineering 133 (1) (2007) 48-58.

口 URL http://ascelibrary.org/doi/abs/10.1061/\%28ASCE\% $290733-9429 \% 282007 \% 29133 \% 3 A 1 \% 2848 \% 29$ 
[19] S. Li, C. J. Duffy, Fully coupled approach to modeling shallow water flow, sediment transport, and bed evolution in rivers, Water Resources Research 47 (3) (2011) W03508.

URL http://dx.doi.org/10.1029/2010WR009751

[20] F. Benkhaldoun, S. Sari, M. Seaid, A flux-limiter method for dam-break flows over erodible sediment beds, Applied Mathematical Modelling 36 (10) (2012) $4847-4861$.

[21] R. Canelas, J. Murillo, R. M. Ferreira, Two-dimensional depth-averaged modelling of dam-break flows over mobile beds, Journal of Hydraulic Research 51 (4) (2013) 392-407. doi:10.1080/00221686.2013.798891.

URL https://doi.org/10.1080/00221686.2013.798891

565 modeling for movable bed flows, Journal of Hydraulic Engineering 142 (12) (2016) 04016069.

[22] X. Liu, J. A. Infante Sedano, A. Mohammadian, A robust coupled 2-D model for rapidly varying flows over erodible bed using central-upwind method with wetting and drying, Canadian Journal of Civil Engineering 42 (8) (2015) 530-543.

URL https://doi.org/10.1139/cjce-2014-0524

[23] X. Liu, A. Mohammadian, A. Kurganov, J. A. Infante Sedano, Well-

a balanced central-upwind scheme for a fully coupled shallow water system modeling flows over erodible bed, Journal of Computational Physics 300 (2015) $202-218$.

URL https://doi.org/10.1016/j.jcp.2015.07.043

[24] F. N. Cantero-Chinchilla, O. Castro-Orgaz, S. Dey, J. L. Ayuso-Muñoz, Nonhydrostatic dam break flows. II: one-dimensional depth-averaged (2016) 04016069 .

URL http://ascelibrary.org/doi/abs/10.1061/\%28ASCE\%29HY. 
[25] G. Nord, M. Esteves, Psem_2d: A physically based model of erosion

n processes at the plot scale, Water Resources Research 41 (8). doi: 10.1029/2004WR003690.

1 URL https://agupubs.onlinelibrary.wiley.com/doi/abs/10.1029/ 2004WR003690

[26] E. Kubatko, J. Westerink, C. Dawson, An unstructured grid mor1 phodynamic model with a discontinuous galerkin method for bed

1 evolution, Ocean Modelling 15 (1) (2006) 71 - 89. doi:https: //doi.org/10.1016/j.ocemod.2005.05.005.

[27] T. Morales de Luna, M. J. Castro-Díaz, C. Parés, E. D. Fernández-Nieto, On a shallow water model for the simulation of turbidity currents, Communications in computational phsics 6 (4) (2009) 848-882. URL https://idus.us .es/xmlui/handle/11441/32102

[28] M. J. Castro-Díaz, E. D. Fernández-Nieto, T. Morales de Luna, G. Narbona-Reina, C. Parés, A HLLC scheme for nonconservative hyperbolic problems. application to turbidity currents with sediment transport ESAIM: M2AN 47 (1) (2013) 1-32.

[29] C. Parés, Numerical methods for nonconservative hyperbolic systems: a theoretical framework, SIAM Journal on Numerical Analysis 44 (1) (2006) 300-321. doi:10.1137/050628052.

URL https://doi.org/10.1137/050628052

${ }_{605}[30]$ Y. Xing, C.-W. Shu, S. Noelle, On the advantage of well-balanced schemes for moving-water equilibria of the shallow water equations, Journal of Scientific Computing 48 (1) (2011) 339-349. doi:10.1007/s10915-010-9377-y URL https://doi .org/10.1007/s10915-010-9377-y 
[31] S. Cordier, M. Le, T. Morales de Luna, Bedload transport in shallow water

[32] G. Parker, Y. Fukushima, H. M. Pantin, Self-accelerating turbidity cur615

[34] F. M. Exner, Zur physik der dünen, Akad. Wiss. Wien Math. Naturwiss, Klasse 129 (1920) 929-952.

[35] S. F. Bradford, N. D. Katopodes, Hydrodynamics of turbid underflows. i: Formulation and numerical analysis, Journal of Hydraulic Engineering 125 (10) (1999) 1006-1015.

URL https://ascelibrary.org/doi/abs/10.1061/\%28ASCE\% $290733-9429 \% 281999 \% 29125 \% 3 \mathrm{~A} 10 \% 281006 \% 29$

[36] R. Zhang, J. Xie, Sedimentation research in China: Systematic selection, China and Water and Power Press, Beijing, 1993.

630 [37] M. Garcia, G. Parker, Experiments on the entrainment of sediment into suspension by a dense bottom current, Journal of Geophysical Research: Oceans 98 (C3) (1993) 4793-4807. doi:10.1029/92JC02404.

[ URL https://agupubs.onlinelibrary.wiley.com/doi/abs/10.1029/ 92JC02404

${ }_{635}^{6}[38]$ Y. Kubo, T. Nakajima, Laboratory experiments and numerical simulation of sediment-wave formation by turbidity currents, Marine Geology 192 (1) 


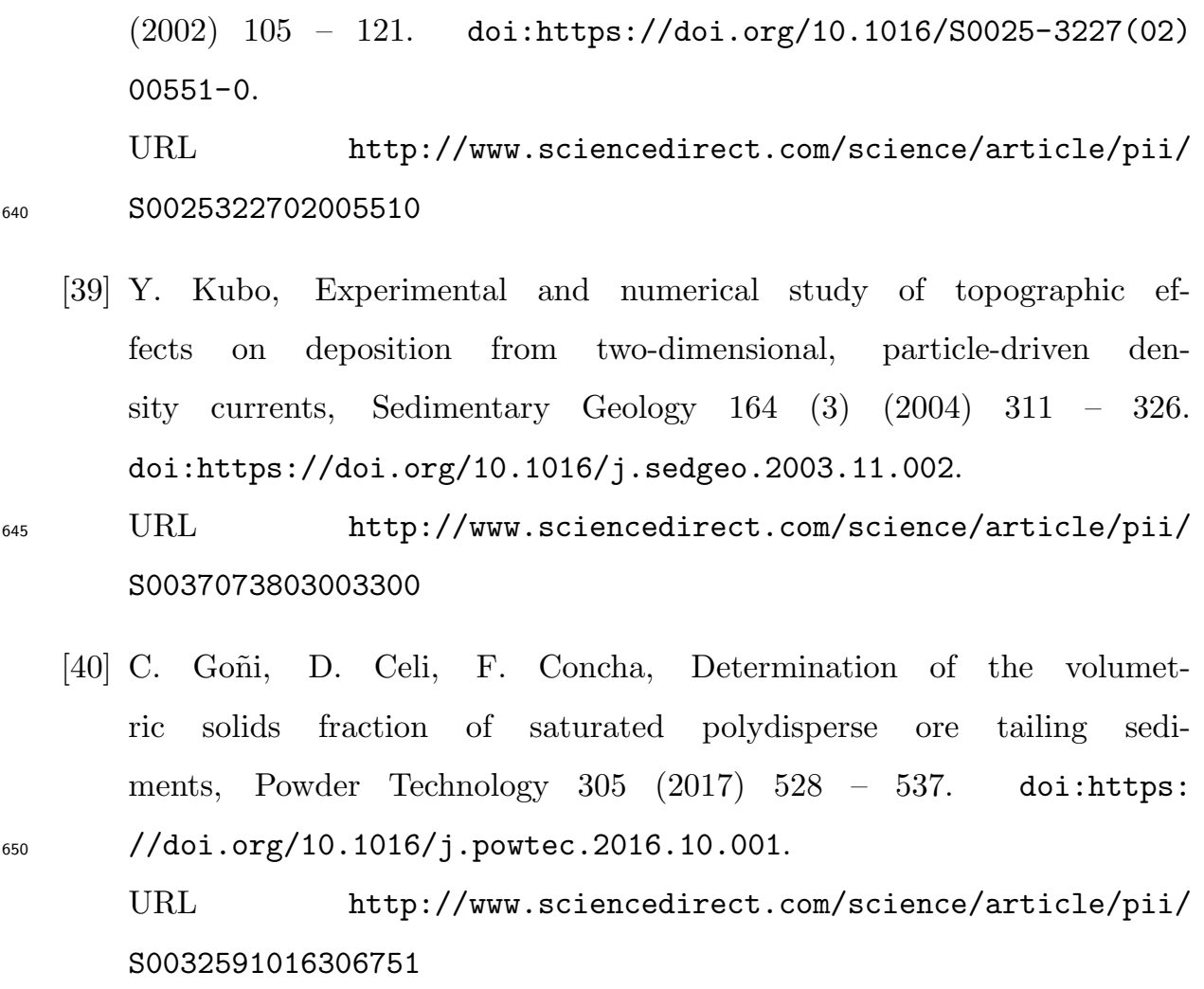

[41] G. Dal Maso, P. G. LeFloch, F. Murat, Definition and weak stability of nonconservative products, Journal de Mathématiques Pures et Appliquées 74 (1995) 483-548.

[42] M. Castro Díaz, E. Fernández-Nieto, A class of computationally fast first order finite volume solvers: Pvm methods, SIAM Journal on Scientific Computing 34 (4) (2012) A2173-A2196. doi:10.1137/100795280.

URL https://doi .org/10.1137/100795280

${ }_{660}^{6}$ [43] P. Degond, P.-F. Peyrard, G. Russo, P. Villedieu, Polynomial upwind schemes for hyperbolic systems, Comptes Rendus de l'Académie des Sciences - Series I - Mathematics 328 (6) (1999) 479 - 483. doi:https://doi.org/10.1016/S0764-4442(99)80194-3.

a URL
S0764444299801943


[44] E. D. Fernández-Nieto, M. J. Castro Díaz, C. Parés, On an intermediate field capturing riemann solver based on a parabolic viscosity matrix for the two-layer shallow water system, Journal of Scientific Computing 48 (1) (2011) 117-140.

685

[48] H. de Vriend, 2dh mathematical modelling of morphological evolutions in shallow water, Coastal Engineering 11 (1) (1987) 1 - 27. doi:https://doi.org/10.1016/0378-3839(87)90037-8

口

690

[49] H. Capart, D. Young, Formation a jump by the dam-break wave over a granular bed, Journal of Fluid Mechanics 372 (1998) 165-187.

URL https://doi .org/10.1017/S0022112098002250 
[50] L. Fraccarrollo, H. Capart, Riemann wave description of erosional dambreak flows, Journal of Fluid Mechanics 461 (2002) 183-238.

URL https://doi.org/10.1017/S0022112002008455

[51] B. Spinewine, Y. Zech, Small-scale laboratory dam-break waves on movable beds, Journal of Hydraulic Research 45 (sup1) (2007) 73-86.

URL https://doi.org/10.1080/00221686.2007.9521834

[52] L. Goutiere, S. Soares-Frazão, Y. Zech, Dam-break flow on mobile bed in abruptly widening channel: experimental data, Journal of Hydraulic Research 49 (3) (2011) 367-371. doi:10.1080/00221686.2010.548969.

URL https://doi.org/10.1080/00221686.2010.548969

[53] C. Swartenbroekx, Y. Zech, S. Soares-Frazão, Two-dimensional two-layer shallow water model for dam break flows with significant bed load transport, International Journal for Numerical Methods in Fluids 73 (5) (2013) 477-508. doi:10.1002/fld.3809.

URL https://onlinelibrary.wiley.com/doi/abs/10.1002/fld.3809

[54] S. Soares-Frazão, R. Canelas, Z. Cao, L. Cea, H. M. Chaudhry, A. D. Moran, K. E. Kadi, R. Ferreira, I. F. Cadórniga, N. Gonzalez-Ramirez, M. Greco, W. Huang, J. Imran, J. L. Coz, R. Marsooli, A. Paquier, G. Pender, M. Pontillo, J. Puertas, B. Spinewine, C. Swartenbroekx, R. Tsubaki, C. Villaret, W. Wu, Z. Yue, Y. Zech, Dam-break flows over mobile beds: experiments and benchmark tests for numerical models, Journal of Hydraulic Research 50 (4) (2012) 364-375. doi:10.1080/00221686.2012.689682.

URL https://doi .org/10.1080/00221686.2012.689682 